\title{
Sliding-window analysis tracks fluctuations in amygdala functional connectivity associated with physiological arousal and vigilance during fear conditioning
}

Article

Accepted Version

Creative Commons: Attribution-Noncommercial-No Derivative Works 4.0

Baczkowski, B. M., Johnstone, T., Walter, H., Erk, S. and Veer, I. M. (2017) Sliding-window analysis tracks fluctuations in amygdala functional connectivity associated with physiological arousal and vigilance during fear conditioning. Neurolmage, 153. pp. 168-178. ISSN 1053-8119 doi:

https://doi.org/10.1016/j.neuroimage.2017.03.022 Available at https://centaur.reading.ac.uk/69567/

It is advisable to refer to the publisher's version if you intend to cite from the work. See Guidance on citing.

To link to this article DOI: http://dx.doi.org/10.1016/j.neuroimage.2017.03.022

Publisher: Elsevier

All outputs in CentAUR are protected by Intellectual Property Rights law, including copyright law. Copyright and IPR is retained by the creators or other copyright holders. Terms and conditions for use of this material are defined in the End User Agreement. 


\section{www.reading.ac.uk/centaur}

\section{CentAUR}

Central Archive at the University of Reading

Reading's research outputs online 
Sliding-window analysis maps amygdala time-varying connectivity

\section{Sliding-window analysis tracks fluctuations in amygdala functional connectivity associated with physiological arousal and vigilance during fear conditioning}

Blazej M. Baczkowski ${ }^{\text {a,b,c,d* }}$, Tom Johnstone ${ }^{\mathrm{e}}$, Henrik Walter $^{\mathrm{a}}$, Susanne Erk ${ }^{\mathrm{a}}$, Ilya M. Veer ${ }^{\mathrm{a}^{*}}$

${ }^{a}$ Department of Psychiatry and Psychotherapy, Charité - Universitätsmedizin Berlin, Campus Mitte, Berlin, Germany

${ }^{\mathrm{b}}$ Max Planck Institute for Human Cognitive and Brain Sciences, Max Planck Research Group for Neuroanatomy \& Connectivity, Leipzig, Germany

${ }^{c}$ Department of Psychology, Leipzig University, Leipzig, Germany

d International Max Planck Research School NeuroCom, Leipzig, Germany

${ }^{\mathrm{e}}$ Centre for Integrative Neuroscience and Neurodynamics, Department of Psychology, University of Reading, Reading, United Kingdom

* To whom correspondence should be addressed.

Word count: 248 (abstract), 7082 (body)

Keywords: functional connectivity, sliding-window analysis, dynamic connectivity, amygdala, fear conditioning

\section{Corresponding Authors:}

Blazej M. Baczkowski, MSc

Max Planck Institute for Human Cognitive and Brain Sciences

Max Planck Research Group for Neuroanatomy \& Connectivity

Stephanstraße 1a, 04103 Leipzig, Germany

Tel. +49341 9940-2431

Emailbbaczkowski@cbs.mpg.de

Ilya M. Veer, PhD

Charité - Universitätsmedizin Berlin, CCM

Department of Psychiatry and Psychotherapy

Division of Mind and Brain Research

Charitéplatz 1, 10117 Berlin, Germany

Tel. +49-(0)30450517223

Emaililya.veer@charite.de 
Sliding-window analysis maps amygdala time-varying connectivity

\section{$1 \quad$ Abstract}

3 We evaluated whether sliding-window analysis can reveal functionally relevant brain network

4 dynamics during a well-established fear conditioning paradigm. To this end, we tested if 5 fMRI fluctuations in amygdala functional connectivity (FC) can be related to task-induced 6 changes in physiological arousal and vigilance, as reflected in the skin conductance level 7 (SCL). Thirty-two healthy individuals participated in the study. For the sliding-window analysis we used windows that were shifted by one volume at a time. Amygdala FC was

9 calculated for each of these windows. Simultaneously acquired SCL time series were 10 averaged over time frames that corresponded to the sliding-window FC analysis, which were 11 subsequently associated with the whole-brain seed-based amygdala sliding-window FC using the GLM. Surrogate time series were generated to test whether connectivity dynamics could have occurred by chance. In addition, results were contrasted against static amygdala FC and sliding-window FC of the primary visual cortex, which was chosen as a control seed, while a physio-physiological interaction (PPI) was performed as cross-validation. During periods of 16 increased SCL, the left amygdala became more strongly coupled with the bilateral insula and medial prefrontal cortex, core areas of the salience network. The sliding-window analysis yielded a connectivity pattern that was unlikely to have occurred by chance, was spatially distinct from static amygdala FC and from sliding-window FC of the primary visual cortex,

20 but was highly comparable to that of the PPI analysis. We conclude that sliding-window 21 analysis can reveal functionally relevant fluctuations in connectivity in the context of an externally cued task. 
Sliding-window analysis maps amygdala time-varying connectivity

2

3

4

\section{Introduction}

(n)

Intrinsic functional connectivity (FC) networks, obtained with fMRI, are not static but can change their configuration considerably at different time scales, ranging from seconds, minutes to hours (Allen et al., 2014; Chang and Glover, 2010; Gonzalez-Castillo et al., 2014; Handwerker et al., 2012; see Hutchison et al., 2013a, for a review; Shen et al., 2015). Changes in resting-state FC observed at longer time scales can be induced by learning (e.g., Lewis et al., 2009; Schultz et al., 2012), and large state transitions such as sleep (e.g., Horovitz et al., 2009; Horovitz et al., 2008), anesthesia (e.g., Boveroux et al., 2010), or stress (e.g., Veer et al., 2011), whereas variations in intrinsic FC at the time-scales of seconds to minutes are detectable within one scanning session (Handwerker et al., 2012; cf., Shehzad et al., 2009; Wang et al., 2012). In contrast to conventional connectivity analysis, in which connections are typically treated as static, techniques investigating non-stationarity of FC have revealed a rich dynamic organization of resting-state networks. These networks tend to segregate and integrate into spatially and temporally overlapping subcomponents over short time intervals (Karahanoglu and Van De Ville, 2015). For example, the default mode network (DMN), which is particularly active during resting-state, includes several functionally distinct and spatially overlapping sub-networks that exhibit their own time-dependent pattern of coupling with other networks (Smith et al., 2012). Zalesky and colleagues (2014) found that most dynamic connections are intermodular, which means that these connections link brain regions from topologically separable modules. Sporadic intervals during which multiple pairs of brain regions spontaneously fluctuate in and out of high correlation over time might be an efficient 
1 mechanism of information exchange between neuronal populations (Zalesky et al., 2014),

2 which, in turn, enables the brain to dynamically integrate and coordinate different neural

3 systems in response to internal and external stimuli across multiple time scales (Hutchison et 4 al., 2013a). Hence, the analysis of time-varying FC has the potential to improve our 5 understanding of brain function. Yet, it remains unclear which techniques are the best for 6 characterizing functionally relevant brain network dynamics with fMRI.

Due to its analytical simplicity, such as computational efficiency and interpretability, sliding-window analysis is the most commonly used strategy for examining time-dependent changes in FC during rest (Chang and Glover, 2010; Hutchison et al., 2013b). Yet, it remains debatable whether the obtained fluctuations in resting-state FC over time with the sliding-

11 window analysis are truly non-stationary, whether these can be attributed to neuronal activity 12 (for a review, see Hutchison et al., 2013a; Preti et al., 2016), whether their statistical estimates are valid and reliable (Betzel et al., 2016; Hindriks et al., 2015; Hlinka and Hadrava, 2015; Zalesky and Breakspear, 2015), and which of its parameters, such as window size or offset, should be used to best characterize connectivity dynamics during resting-state when the timing, duration, and composition of transient FC patterns are unknown (Leonardi and Van De Ville, 2015; Shakil et al., 2016). To address some of these challenges, previous studies investigated the performance of the sliding-window analysis with simulated and empirical data linked to behavioral measures or simultaneously acquired electrophysiology.

Studies that used simulated data identified statistical pitfalls in the assessment of time21 varying connectivity retrieved with the sliding-window analysis and analyzed the 22 performance of the method in characterizing FC dynamics. For example, Hindriks and 


\section{Sliding-window analysis maps amygdala time-varying connectivity}

1 colleagues (2015) pointed out that the mere presence of fluctuations in FC observed with the

2 sliding-window analysis is not evidence of connectivity dynamics, and emphasized the

3 importance to test against a null distribution of surrogate data when inferring time-varying

4 connectivity. The method's performance strongly depends on window length, which should

5 be large enough to permit robust estimation of FC, yet small enough to detect potentially

6 interesting transient fluctuations in FC. Window lengths between 30-60 sec seem favorable

7 (Allen et al., 2014; Hutchison et al., 2013b; Jones et al., 2012; Liegeois et al., 2016; Shirer et al., 2012; Yang et al., 2014), but the exact duration of transiently stable FC patterns remains

9 rather unknown and might change with task demands. Shakil and colleagues (2016) showed

10 that window size and offset may have the biggest impact on the accuracy of the results,

11 followed by such factors as noise levels and temporal filtering. In addition, the frequency components of the fMRI signal tend to interact with the size of the window, and produce spurious fluctuations of sliding-window connectivity (Leonardi and Van De Ville, 2015).

Such spurious fluctuations can be avoided by first applying a high-pass filter to the original time series (i.e., before windowing) with a cut-off frequency of $1 / w$, where $w$ represents the 16 length of the window in seconds.

For empirical data, the output of the sliding-window analysis is typically fed into a clustering algorithm to retrieve transient FC patterns, which have their specific temporal and spatial features, and which are presumed to reflect cognitive operations. With this procedure empirical research showed that intrinsic FC alternates between a finite number of transient 21 connectivity patterns, that emerges and dissolves over short periods of time (Allen et al., 2014). Such transient FC patterns can be characterized in terms of their temporal and spatial 


\section{Sliding-window analysis maps amygdala time-varying connectivity}

1 characteristics. For example, their temporal characteristics tend to differentiate between

2 groups (in case of schizophrenia patients, see Damaraju et al., 2014). Spatially, regions that

3 exhibit functional diversity tend to participate in different transient FC patterns. Such

4 association was previously observed for the posteromedial cortex, a key region in the DMN:

5 The subdivisions of the posteromedial cortex take on different transient FC patterns, and each

6 subdivision has its own preferred connectivity state (Yang et al., 2014). Interestingly, the

7 percentage of time that each of these subdivisions spend in a given FC state could be

8 associated with behavioral performance based on executive functions. While analyses of

9 transient FC states that occur during resting-state do provide information about intrinsic

10 connectivity dynamics, such analyses typically assume the presence of distinct FC states.

11 Sharp transitions between such states, however, may be less likely to occur in the brain than 12 more gradual changes in FC dynamics.

The gradual changes can be tracked with concurrent independent measurements, such as simultaneously acquired electrophysiological (e.g., EEG) or peripheral physiological (e.g., heart rate) data. Simultaneous recordings of fMRI and EEG showed that variations in FC are

16 to some degree of neuronal origin and perhaps linked with changes in cognitive or vigilance state (Chang et al., 2013a; Tagliazucchi et al., 2012). In a similar vein, Chang and colleagues (2013b) demonstrated that intrinsic amygdala sliding-window FC with the brainstem, thalamus, putamen, and dorsolateral prefrontal cortex is associated with changes in autonomic state, as indexed by temporal dynamics of heart rate variability. Yet, heart rate variability can

21 contribute directly to the measured BOLD signal through hemodynamic and pulsatile effects 22 (Birn, 2012; Chang et al., 2009), so it is uncertain whether measured FC dynamics reflect 
1 neural coupling or these other, potentially confounding, factors. Although these studies

2 informed us about (neuro-)physiological contribution to fMRI connectivity dynamics revealed

3 by sliding-window analysis, one could only speculate whether the obtained dynamics are

4 functionally relevant. Resting-state indeed seems to reflect the functional architecture of the

5 brain (Sadaghiani, Kleinschmidt, 2013), as it tends to maintain the connectivity dynamics

6 induced by a behavioral context (e.g., Lewis et al., 2009; Tambini et al., 2010). Yet, it

7 remains unclear whether sliding-window analysis is able to capture such functionally relevant

8 connectivity dynamics. Hence, it might be crucial to first indicate that sliding-window FC is

9 related to cognitive processing during a well-described cognitive task.

10 In the current study we therefore investigated whether the sliding-window analysis is

11 able to capture cognitively relevant fluctuations in FC induced by a task. For this purpose we 12 tested whether connectivity dynamics during a well-established classical fear conditioning 13 paradigm, measured with a sliding-window analysis, can be associated with task-induced 14 changes in physiological arousal and vigilance, as reflected in the skin conductance level (SCL). Simultaneous acquisition of SCL during the task enabled us to look at gradual changes in FC associated with cognitive processing that is typically involved in the acquisition of fear. We used the amygdala as an a priori seed for the FC analysis, because it is crucial for the acquisition of conditioned fear responses, including modulation of autonomic function 19 (Phelps and LeDoux, 2005), such as skin conductance. Fear conditioning is typically marked 20 by the gradual increase in the skin conductance response to a neutral stimulus (the 21 conditioned stimulus, CS) that is repetitively paired with an aversive stimulus (the unconditioned stimulus, UCS, typically an electric shock), which in turn leads to changes in 
1 the SCL over the course of the learning (Delgado et al., 2006; Phelps et al., 2004). In parallel,

2 fear conditioning leads to changes in amygdala activation (LaBar et al., 1998), and its FC with

3 the medial prefrontal cortex (Kim et al., 2011; Liu et al., 2011). Thus, simultaneously

4 acquired SCL reflects changes in physiological arousal and vigilance (Boucsein, 2012), which

5 are likely associated with changes in amygdala activity and amygdala-prefrontal coupling that

6 occur during fear conditioning (Davis, 1992; LeDoux, 2000). We expected that sliding-

7 window FC between the amygdala and medial prefrontal cortex would be associated with the

8 fluctuations in SCL, which in turn would indicate connectivity dynamics involved in the

9 acquisition of fear.

2. Methods and materials

\subsection{Participants}

16 Thirty-four healthy German volunteers were recruited in Bonn to take part in a study on fear conditioning and extinction, which comprised a two-day scanning session. Its results were previously published elsewhere (Bilkei-Gorzo et al., 2012). Two participants were excluded

19 from the analyses: imaging data (fMRI) of one participant were unavailable, and 20 physiological data (skin conductance level) of another participant lacked fluctuations over 21 time. The final sample comprised thirty-two participants ( 15 female, mean age=26 years, 22 $\mathrm{SD}=5.59)$ 
Sliding-window analysis maps amygdala time-varying connectivity .

None of the participants had a lifetime diagnosis or a family history of affective disorders. All participants gave written informed consent, and the study was approved by the local ethics committee of the University of Bonn.

\subsection{Study design and procedure}

The original study involved a task for the acquisition and extinction of fear responses that was based on a partial reinforcement learning paradigm (Phelps et al., 2004). The task led to significant activation in brain regions responsible for fear memory formation and extinction, as well as to significant changes in its physiological markers (i.e., skin conductance response), which was presented in the original report (Bilkei-Gorzo et al., 2012).

For the purpose of this study, we used the data of the fear conditioning phase only. In this phase, participants were exposed to a mildly painful laser shock to the foot and colored squares (blue and green), which played a role as aversive unconditioned stimulus (UCS) or conditioned stimuli (CS), respectively. One of the colored squares $(\mathrm{CS}+)$ was partly paired with the UCS, while the other one (CS-) was never paired with the UCS. Participants were exposed to the same sequence of 20 presentations each of the CS+ and CS- in a pseudorandomized order. Ten additional $\mathrm{CS}+$ presentations co-terminated with the UCS. This was a continuous task, in which every stimulus presentation lasted $4 \mathrm{~s}$ and an inter-stimulus interval was set to $12 \mathrm{~s}$ to allow the skin conductance to recover in between trials. After the acquisition phase, participants rated the intensity of the pain stimulus on a 10-point Likert scale (0-not painful at all, 10-unbearable pain). Before the start of the study participants were told that 
Sliding-window analysis maps amygdala time-varying connectivity

1 they would see blue and green colored squares, and that it might be possible that during the

2 presentation of either the blue or the green square a painful stimulus would be applied on their 3 left foot.

\subsection{Imaging data acquisition and preprocessing}

6

Anatomical and functional imaging data were acquired with a Phillips Achieva 3T scanner at the Department of Radiology, University of Bonn. A standard high-resolution T1-weighted anatomical scan (1mm isotropic voxels) was acquired for registration purposes. Functional images were acquired with a $2^{*}$-weighted gradient-echo echo-planar imaging (EPI) sequence using parallel imaging (sensitivity encoding, SENSE factor 2) with the following scan parameters: 413 volumes, 39 axial slices, $3 \times 3 \times 2.4 \mathrm{~mm}$ voxels, $0.6 \mathrm{~mm}$ slice gap, $\mathrm{TR}=1960 \mathrm{~ms}$, $\mathrm{TE}=30$, flip angle $=80^{\circ}, \mathrm{FoV}=192 \mathrm{~mm}(64 \times 64$ matrix $)$. FMRI data processing was carried out using FEAT (FMRI Expert Analysis Tool) Version 5.98, part of FSL (FMRIB's Software Library; Smith et al., 2004). The following pre-statistics processing was applied: motion correction (MCFLIRT, Jenkinson et al., 2002), non-brain tissue removal, spatial smoothing using a $6 \mathrm{~mm}$ full-width at half-maximum Gaussian kernel, grand-mean intensity normalization of the entire 4D dataset by a single multiplicative factor. None of the participant exhibited motion that was greater than $3 \mathrm{~mm}$ (translation) and $1^{\circ}$ (rotation), and the average frame-wise displacement was below 0.3 . We used MELODIC 3.0 as a part of FSL package to decompose each single subject 4D dataset into different spatial and temporal components using Independent Component Analysis 
Sliding-window analysis maps amygdala time-varying connectivity

1 (ICA). ICA components were manually classified as ones representing data artifacts from

2 head motion, cerebrospinal fluid, white matter, and cyclic cardiac and respiratory noise,

3 which were subsequently removed from the 4D dataset (cf., Salimi-Khorshidi et al., 2014).

4 The cleaned data were then filtered using a high-pass temporal filter of $0.025 \mathrm{~Hz}$. The filter

5 cut-off was determined by the window length of 39.2s (i.e., 20 volumes), which was used in

6 the sliding-window analysis (see below), because the transient fluctuations of functional

7 connectivity are limited to the cut-off frequency of the window length (Leonardi and Van De

8 Ville, 2015). We carried out registration of the single subject 4D data to the high-resolution

9 T1-weighted image, and the $\mathrm{T} 1$ to the $2 \mathrm{~mm}$ isotropic MNI-152 standard space image (T1

10 standard brain averaged over 152 subjects; Montreal Neurological Institute, Montréal, QC,

11 Canada). The resulting transformation matrices were then combined to obtain a native to MNI space transformation matrix and its inverse (MNI to native space).

\subsection{Physiological data acquisition and preprocessing}

Skin conductance was recorded during the fMRI acquisition using $\mathrm{Ag} / \mathrm{AgCl}$ electrodes attached to the palmar surface of the left index and middle finger, and the signal was amplified and digitalized via a skin conductance processing unit (Nexus-16, Mind Media). The signal was recorded digitally at the sampling rate of $100 \mathrm{~Hz}$ using Biotrace software (Mind Media), and then resampled to $32 \mathrm{~Hz}$. We preprocessed skin conductance level (SCL)

21 time series with a low-pass filter of $2 \mathrm{~Hz}$, which is a standard procedure (Boucsein et al., 22 2012). 
Sliding-window analysis maps amygdala time-varying connectivity

$1 \quad 2.5$ Sliding-window functional connectivity and statistical analyses

2

3 Seeds of the left and right amygdala were created with the Harvard-Oxford Subcortical 4 Structural Probability Atlas (80\% probability) provided by FSL (Figure S1a). The left and

5 right amygdala were used separately, because lateralization effects have been reported in the

6 fear conditioning literature (Baker and Kim, 2004; van Well et al., 2012), and have been

7 found in the original report of the study as well (Bilkei-Gorzo et al., 2012). To assess time-

8 varying $\mathrm{FC}$, the fMRI datasets and amygdala time series were partitioned into rectangular

9 windows with a length of 39.2 s (i.e., 20 volumes), well within range of a window length

10 between 30-60 s, which has been considered feasible previously (Jones et al., 2012). The

11 windows were shifted by one volume at a time ( $98 \%$ overlap between adjacent windows).

12 Next, amygdala FC was determined for each window by regressing (using the GLM, as

13 implemented in the FSL tool "fsl_glm") the amygdala time series against the time series of all

14 other voxels. FC maps of all windows were then temporally concatenated to create a single

15 4D connectivity data set.

16 Four steps were undertaken to evaluate whether sliding-window analysis can reveal 17 functionally relevant amygdala connectivity dynamics during the fear conditioning paradigm.

18 First, to evaluate the functional relevance of patterns of amygdala FC change over time,

19 simultaneously acquired skin conductance level (SCL) time series were resampled to match

20 the TR of the fMRI data, and subsequently averaged over time frames that corresponded to

21 the windowed time series of amygdala FC. Associations between fluctuations in SCL and 22 amygdala sliding-window FC were assessed using the GLM, and finally tested across the 


\section{Sliding-window analysis maps amygdala time-varying connectivity}

1 group using non-parametric statistics, as implemented in the FSL tool "randomise" (5000

2 permutations; Winkler et al., 2014). To avoid an arbitrarily selected cluster-size forming

3 threshold, and to enhance areas of signal that exhibit some spatial contiguity in the group4 level statistical map, we used the threshold-free cluster enhancement (TFCE) algorithm, and 5 subsequently corrected the results for multiple comparisons at $p<.05$ by using the null 6 distribution of the maximum TFCE value across the map (Smith and Nichols, 2009). In the 7 group-level analysis, we used a mask of the minimum whole brain coverage across all 8 participants (Figure S2).

Second, to provide evidence for non-stationarity of the connectivity results obtained 10 with the sliding-window analysis and its association with SCL (see Section 3.2), we 11 performed additional analyses with surrogate data (cf. Hindriks et al., 2015). To obtain surrogate time series, we used Amplitude Adjusted Fourier Transformed (AAFT) algorithm implemented in MATLAB as an open-source function (Barnett, 2016), which preserves the amplitude, mean, variance, and the amount of auto-correlation of the original time series (Kugiumtzis, 2000). We restricted this analysis to connectivity between the amygdala and a spherical region of interest (ROI) of $6 \mathrm{~mm}$ radius centered around the peak significant voxel (resulting from the first analysis) in the anterior cingulate cortex (ACC; MNI coordinates: 4, 32, 18; see Figure S1b). The BOLD time series from the left amygdala seed and this ACC ROI were extracted for each individual. Then we generated 1000 AAFT surrogate BOLD time series of the left amygdala and ACC ROI, and computed sliding-window FC between 21 them. Subsequently, to test whether the variability of the sliding-window FC (left amygdalaACC ROI) is higher than could be expected by chance, the standard deviation of the obtained 


\section{Sliding-window analysis maps amygdala time-varying connectivity}

1 sliding-window connectivity time series was computed per iteration. As a result, a null

2 distribution of group averaged standard deviation values was obtained (Figure S3a), and used

3 to test whether the group averaged standard deviation of the true left amygdala-ROI sliding-

4 window time series is a random draw from the null distribution. Additionally, to test whether

5 the association between amygdala sliding-window FC with SCL was merely found by chance,

6 sliding-window connectivity time series between the left amygdala and the ACC ROI were

7 extracted for each individual, and then fed into the AAFT algorithm to generate 1000

8 surrogate time series of sliding-window FC. The surrogate time series were subsequently used

9 in the GLM with the true individual SCL time series as a predictor. A group averaged $t$ value

10 was computed per iteration. As a result, a null distribution of group averaged $t$ values

11 expressing the temporal association between the SCL time series and surrogate slidingwindow FC was obtained (Figure S3b). We then tested the null hypothesis that the true observed group averaged $t$ value would be a random draw from this null distribution.

Third, we assessed the spatial specificity of the sliding-window amygdala connectivity. Initially, to explore whether sliding-window amygdala connectivity deviates spatially from its typical static representation (cf. Betzel et al., 2016), we performed a standard whole-brain seed-based connectivity analysis across the whole task scan for the left amygdala. A voxel-wise FWE threshold of $p<.05$ was used to identify a spatial pattern of FC across participants, which exhibits the strongest temporal association of the amygdala. Note that TFCE-based correction was not used for this analysis, as the input images (individual amygdala connectivity maps) mostly contained positive values. In this case, TFCE fails to produce informative results, as it will produce significance across the whole brain. 


\section{Sliding-window analysis maps amygdala time-varying connectivity}

1 Subsequently, to determine whether the observed pattern of fluctuations in FC is specific for

2 the amygdala rather than due to unlocalized global activity (Scholvinck et al., 2010), we

3 performed additional analysis for a control region. Specifically, to better disentangle the

4 effects of global vs. induced arousal measured with SCL, we chose a region that has a high

5 signal-to-noise ratio (SNR), and should be activated by the task. The primary visual cortex

6 fulfilled both these criteria, as it typically shows a high SNR and should be activated by the

7 presentation of visual stimuli in the task, which were sometimes paired with the electric

8 shock. The mask for the seed in the visual cortex was derived from a template of the primary

9 visual cortex by Shirer and colleagues (2012). The template (Functional Imaging in

10 Neuropsychiatric Disorders [F.I.N.D.] Lab, 2016) was eroded by a kernel of 4mm-radius-

11 sphere, yielding our seed mask of the primary visual cortex seed (Figure S1c).

Fourth, to further test whether the temporal association between the amygdala FC changes and SCL obtained with the sliding-window analysis are of functionally relevance, we performed a PPI analysis, which is the conventional method to study connectivity changes over time when changes in physiology (or task conditions) are known (Friston et al., 1997;

16 Prado, Weissman, 2011). Although we did not model task predictors in our analysis, SCL can 17 be used as an indirect model of task-related response, and hence be used as a predictor for FC changes in the PPI analysis. If its results were to be comparable with the results of the slidingwindow analysis, this would increase confidence that the sliding window approach is able to capture FC dynamics in the context of our task. To this end, we resampled the preprocessed

21 SCL time series to the total number of volumes in the scanning session (i.e., 413). The skin conductance impulse response function resembles the canonical hemodynamic response 
Sliding-window analysis maps amygdala time-varying connectivity

1 function very closely in its shape and latency (Bach et al., 2009; Lim et al., 1997) and thus

2 SCL could be cross-correlated with BOLD signal without additional processing (i.e., without

3 convolution with a hemodynamic response function). The GLM was conducted with PPI

4 regressors of: (1) main effect of the seed regressor, (2) main effect of SCL time series

5 regressor, and (3) the interaction of the seed (demeaned) and SCL regressor (centered), which

6 was treated here as the regressor of interest, and which was used in the second level random

7 effects analysis performed with the same non-parametric statistics as used for the sliding

8 window analysis (5000 permutations; $p<.05$, TFCE corrected for multiple comparisons).

Lastly, we assessed the association between fluctuations in BOLD activity and SCL

10 across the whole task scan to compare the results of sliding-window connectivity with mere

11 fluctuations in BOLD. To this end, we used SCL, resampled to the number of TRs, as a 12 predictor in the GLM, and examined both its positive and negative associations for each 13 participant separately. The resulting individual statistical maps were then subjected to a 14 second level random effects analysis with the same parameters as were used above (5000 15 permutations; $p<.05$, TFCE corrected for multiple comparisons). 
Sliding-window analysis maps amygdala time-varying connectivity

\section{Results}

\subsection{Fluctuations in skin conductance level and presentation of electric shocks}

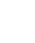

Standard analysis of skin conductance response, time-locked to the presentation of electric shocks was previously reported in the original study. To indicate the most representative time course across the group of participants, we ran a principal component analysis (PCA) on the set of individual SCL time series. Figure 1a depicts the first eigen-time-series of SCL across the entire task scan, in which the red vertical lines indicate the timing of electric shocks. SCL typically rises after the shock and tends to recover in between consecutive shocks. Taken together, these results indicate that the fear conditioning task, and especially the presentation of shocks, affected the fluctuations of SCL.

[Figure 1 about here]

\subsection{Sliding-window analysis of amygdala FC and SCL}

To investigate the temporal relationship between amygdala FC and fluctuations in SCL during fear conditioning, we employed a whole brain seed-based sliding-window FC analysis with $98 \%$ overlap between two consecutive windows (i.e., shifting the window by one volume at a time). The fluctuations in SCL significantly co-varied with the fluctuations in left amygdala FC obtained with the sliding-window analysis. We identified a set of regions comprising the 
1 bilateral insula and medial prefrontal cortex (mPFC), reminiscent of the salience network

2 (Seeley et al., 2007), which became more strongly coupled with the left amygdala during 3 periods of increased SCL (Figure 2a; Table 1). We did not find any significant result for the 4 right amygdala at the corrected threshold of $p=.05$. Even the uncorrected map, liberally 5 thresholded at $p=.01$ did not show the clear pattern of the right amygdala. No negative 6 associations were observed for the left or right amygdala.

7
[Table 1 about here]

\section{[Figure 2 about here]}

To explore the most representative pattern of fluctuations in sliding-window connectivity across the group of participants, we performed a PCA on the individual sliding window connectivity time series between the amygdala and the ACC ROI that showed the strongest effect. Figure $1 \mathrm{~b}$ depicts the first eigen-time-series of the sliding-window connectivity, with vertical red lines (roughly) indicating the time points of electric shocks. Overall, there seems to be good correspondence between exposure to shocks and increases in the amygdala-ACC sliding-window connectivity.

\subsection{Assessment of non-stationarity}

To determine non-stationarity of the connectivity time series obtained with the sliding- 
1 window analysis, we calculated 1000 surrogate BOLD time series for the left amygdala and

2 ACC ROI (cf. Hindriks et al., 2015). Per iteration the standard deviation of the time-varying

3 connectivity between the two surrogate time series was computed. As a result, a null

4 distribution of group averaged standard deviation values was obtained (Figure S3a), and used

5 to test whether the group averaged standard deviation of the true left amygdala-ROI sliding-

6 window time series is a random draw from the null distribution. The true observed group

7 averaged value of the standard deviation was 0.265 , and its probability under the null

8 distribution was $p=.024$. This result suggests that the variability of the sliding-window FC

9 during our task is unlikely to have emerged simply by chance, and could thus be considered

10 non-stationary.

11 Secondly, to test the validity of the association between amygdala sliding-window FC

12 with SCL, we generated a null distribution of group averaged $t$ values expressing the temporal

13 association between the true SCL time series and a 1000 surrogate sliding-window FC time

14 series (Figure $\mathrm{S} 3 \mathrm{~b}$ ). We tested the null hypothesis that the true observed group averaged $t$

15 value would be a random draw from this null distribution. The true observed value was $t=$

163.74 , and its probability under the null distribution is $p<.001$. This result suggest that the temporal association between the fluctuations in left amygdala FC and SCL, as obtained with the sliding-window analysis, is in fact dependent on the sequence of events in time, rather than reflecting general low frequency fluctuations that are unspecific to the task. 
Sliding-window analysis maps amygdala time-varying connectivity

\section{$1 \quad 3.4$ Spatial specificity of the sliding-window amygdala results}

2

3 The pattern of static amygdala connectivity is in line with what was reported previously (e.g.,

4 Veer et al., 2011; Roy et al., 2009), although only positive connectivity values were found 5 here, and qualitatively different for the pattern that emerged from the sliding-window analysis 6 (see the figure S4). This suggests that the sliding-window connectivity of the amygdala associated with SCL is spatially distinct from the static (averaged) FC of the amygdala.

Next, we examined whether we could observe similar associations between SCL and time-varying FC for the primary visual cortex, an area that was not expected to mediate the effects of fear conditioning present in changes of SCL. We found several regions that exhibited a positive association between the fluctuations in primary visual cortex slidingwindow connectivity and SCL. In comparison to the results of the sliding-window analysis of the left amygdala, FC of the primary visual cortex seemed to increase with a more dorsal part of the left mPFC, superior PFC, and caudate during periods of elevated SCL (Figure 1b). However, a formal statistical test only demonstrated this differentiation at a lenient uncorrected threshold $(p<.05)$.

\subsection{Physio-physiological analysis}

To test whether the results obtained with the sliding-window analysis are comparable to a more standard method to test for connectivity changes as a function of SCL, we performed a PPI analysis. Again, we restricted our analysis to the left amygdala. We found only one 
1 cluster that showed significant results (right anterior insula: peak coordinates $\mathrm{MNI}=46,14$, -

$212 ; p=.036$; cluster size $=24$ voxels $)$. However, at a liberal uncorrected threshold $(p<.01)$

3 the PPI analysis demonstrated a connectivity pattern similar to the sliding-window analysis

4 (Figure 3). This finding does not only suggest that sliding-window analysis may be a feasible

5 method to capture transient and functionally relevant changes in FC during a task, but also

6 suggest that it may even be more sensitive than the commonly used PPI analysis to reveal

7 time-varying connectivity.

8 relation with amygdala sliding-window functional connectivity.

[Figure 3 about here] scan

\subsection{Association between SCL and fluctuations in BOLD activity across the whole task}

We did not observe any significant results for the positive contrast at a whole-brain corrected threshold. However, only at the very lenient uncorrected threshold of $p=.05$ we found clusters in the bilateral anterior insula. The negative contrast did show an association, which survived correction for multiple comparisons and is depicted in figure S5. These findings suggest that SCL alone is associated with a qualitatively different pattern of brain activation than its 
Sliding-window analysis maps amygdala time-varying connectivity

2

4

5

\section{Discussion}

(

In the present study we set out to test whether sliding-window analysis could reveal behaviorally relevant time-varying connectivity fluctuations. To this end, sliding-window amygdala functional connectivity (FC) was assessed during a well-established fear conditioning paradigm, and was linked to changes in physiological arousal and vigilance, as reflected by changes in skin conductance level (SCL). During periods of increased SCL, the left amygdala became more strongly coupled with the bilateral insula and medial prefrontal cortex, most notably the anterior cingulate cortex (ACC). This result was unlikely to have emerged merely by chance, as it was not present in the surrogate data. It was spatially distinct from the static left amygdala FC and from sliding-window FC of a control seed in the visual cortex. FC of the right amygdala did not exhibit fluctuations associated with changes in SCL. Furthermore, the more commonly used physio-physiological interaction (PPI) analysis demonstrated a connectivity pattern of the left amygdala that was similar to the slidingwindow analysis, albeit only at a liberal, uncorrected threshold.

With the sliding-window analysis, we identified a specific connectivity pattern that is associated with gradual changes in SCL, and spatially distinct from its static (averaged) connectivity (Roy et al., 2009; Veer et al., 2011). Skin conductance is typically used to quantify both tonic and phasic arousal (Boucsein, 2012). In the current study, we mostly captured the tonic component, on top of which phasic changes occurred that were elicited by the stimulus presentations. Previously, such changes were associated with vigilance performance in attention demanding tasks, and with the level of arousal elicited by either 
Sliding-window analysis maps amygdala time-varying connectivity

1 positive or negative pictures. Hence, we attribute the changes in SCL to changes in 2 physiological arousal and vigilance induced by the presentation of shocks.

SCL was associated with a qualitatively different pattern of fluctuations in BOLD activity across the whole task scan than its association with amygdala sliding-window FC, which comprised regions of the salience network (Seeley et al., 2007). This network is typically associated with the detection of behaviorally salient events and has been implicated in dynamic switching between the DMN and the central executive network (Chen et al., 2013; Goulden et al., 2014; Jilka et al., 2014), which can be tracked by a physiological index of arousal (i.e. heart rate; Young et al., 2016). The salience network was found to be activated during fear conditioning tasks (Sehlmeyer et al., 2009) and pain perception (Garcia-Larrea 11 and Peyron, 2013). Notably, the ACC and parts of the bilateral insula seemed strongly coupled with the left amygdala during the periods of increased SCL. This in contrast to the connectivity profile of the primary visual cortex, which connectivity changes were likely induced by the presentation of visual stimuli in the experiment. These results suggest that the sliding-window method captured time-varying FC of the left amygdala related to distinct cognitive processing involved in the acquisition of a fear response. Specifically, FC between

17 the amygdala and insula seems to be associated with regulating vigilance (Denny et al., 2014; Stein et al., 2007), whereas FC between the amygdala and medial PFC is typically involved in 19 the regulation of autonomic states, such as skin conductance and cardio-vascular activity 20 (Etkin et al., 2011; Gianaros et al., 2008; Wager et al., 2009). Ongoing cardio-vascular 21 parasympathetic activity was previously associated with the fluctuations in the strength of the amygdala-mPFC coupling while individuals were watching sadness-inducing film excerpts 


\section{Sliding-window analysis maps amygdala time-varying connectivity}

1 (Raz et al., 2012). Therefore, we infer that the observed connectivity changes were likely

2 driven by the task, which influenced amygdala-insula FC due to an increased, but unknown

3 probability of receiving an electric shock (vigilance), as well as amygdala-mPFC coupling

4 due to the presentation of the shock itself (autonomic regulation). This was corroborated by

5 the seemingly good overlap between shock exposure and increases in both SCL and amygdala

6 sliding-window connectivity. In contrast, we did not find the effect in the right amygdala,

7 despite its involvement in fear extinction (LaBar et al., 1998), pain (Ji and Neugebauer,

8 2009), and processing of negative emotions (Angrilli et al., 1996) has been well established.

Previous studies used simulated data to evaluate the performance of the method, and

10 provided guidelines for the choice of its parameters, and for how to carry out hypothesis

11 testing. Following these guidelines, we evaluated the performance of sliding-window analysis in empirically acquired task data, for which the neural correlates have been well-described before. We found that sliding-window analysis was able to detect variation in FC over time associated with changes in SCL, which were (in part) evoked by the fear conditioning paradigm. Importantly, these changes were significantly different from those obtained with

16 surrogate data. In a previous study, however, sliding-window derived fluctuations in FC across time that was based on randomly shuffled data resembled the variability of nonshuffled data quite well (Hindriks et al., 2015), which questioned the validity of the method in revealing true connectivity dynamics. Although direct comparison of their and our findings is

20 hampered by the different nature of the data (resting-state vs. task-based condition), similarly 21 to Hindriks and colleagues we emphasize that a statistical description of sliding-window 22 fluctuations alone is not sufficient to reveal its functional relevance, but only becomes 


\section{Sliding-window analysis maps amygdala time-varying connectivity}

1 informative when supported by associations with a concurrent behavioral or physiological

2 measure. That is, in our study, to give meaningful interpretation of potentially insignificant

3 connectivity fluctuations, we used simultaneously acquired SCL, which enabled us to validate

4 the time-course of such connectivity fluctuations against another task-derived measure.

5 Therefore, for future studies we would like to stress the importance of collecting a behavioral

6 (and/or physiological) measure, which could be used as a temporal model, and thus as an

7 external validation, for studying gradual network dynamics associated with changes in 8 cognition.

Lastly, we demonstrated that traditional PPI analysis was able to reveal a similar 10 pattern of time-varying connectivity as the sliding-window, albeit only at a liberal, 11 uncorrected threshold, which provides convergent validity for our findings. Moreover, it seemed that more robust results were generated by the sliding-window analysis. We propose a twofold interpretation of these results: (i) sliding-window FC did show a cognitively-relevant pattern of fluctuations that is typically captured by PPI analysis on task-based fMRI data; (ii) sliding-window analysis may ultimately generate more sensitive results, as it captures the overall effect of the task, whereas PPI analysis isolates the effects of the seed, the task (i.e., the SCL in the current analysis), and the interaction, which might lack efficiency when one of the two main regressors is correlated with the interaction term (Friston et al., 1997). Yet, the potential higher sensitivity of the sliding-window compared to PPI should be investigated in a range of task contexts, including the test-retest reliability of the two methods. This would give

21 more confidence about whether the sliding-window method truly can be deemed more robust, and could exclude the alternative possibility that the lower $p$ values obtained with the PPI 
Sliding-window analysis maps amygdala time-varying connectivity

1 analysis may speak for its selectivity rather than less sensitivity.

2

\subsection{Limitations and possible improvements}

We used SCL as a non-vascular index of autonomic activity that should be less intertwined

6 with BOLD than cardiovascular measures, such as heart rate variability (e.g., Chang et al., 2013). Yet, both SCL and cardiovascular measures are important markers of autonomic nervous system activity, and hence are not easy to treat as independent measures. Indeed it is likely that changes in SCL are accompanied by changes in cardiovascular measures and/or respiration. Unfortunately, none of theses additional autonomic indices (ECG/pulse oximeter, respiration) were acquired, thus we could not test to what extent SCL is collinear with these types of data.

In this study we did not only take advantage of employing simultaneously acquired SCL as a temporal model of connectivity changes, but also of incorporating task conditions. While the task paradigm enabled us to infer whether the observed connectivity changes associated with SCL reflect cognitive processing, in this case threat appraisal, one must acknowledge that transient co-activation in response to external stimulation can be interpreted as functional connectivity, even when no information exchange might exist. Nevertheless, we believe that the dynamics we observed between the amygdala and regions of the salience network do reflect meaningful "cross-talk" between these regions. The existing literature

21 shows consistent involvement of the amygdala and the salience network in fear conditioning, which orchestrated activity creates a state of readiness to respond to potential threat (Fullana 


\section{Sliding-window analysis maps amygdala time-varying connectivity}

1 et al. 2016; Phelps et al. 2001). It is more likely that transient and consistent task induced co-

2 activation truly reflects information exchange if the regions involved are anatomically

3 connected as well. Both primate and human studies show that the amygdala has a vast number

4 of anatomical projections to the medial PFC and insula (Barbas and De Olmos 1990; Amaral

5 and Price 1984; Ghashghaei and Barbas 2002; Kim et al. 2011; Fani et al. 2015; Greening and

6 Mitchell 2015), which enables the functional integration that contributes to fear processing

7 and regulation (Stein et al. 2007; Liu et al. 2011; Wheelock et al. 2014; Vytal et al. 2014).

8 Persistent activity of the network was observed in the aftermath of the fear conditioning,

9 which further suggests its involvement during the acquisition of fear (Feng et al. 2014).

10 Lastly, if the synchronization between the amygdala and the salience network was only due to

11 external stimulation, it should be present among all regions involved in the task. While the

12 seed in the visual cortex was involved in the task and its activity coincided with the task

13 stimulation, it showed a qualitatively different spatial pattern of connectivity changes with

14 SCL. This further corroborates our interpretation of information exchange between the 15 amygdala and the salience network, instead of having found mere co-activation.

16 The task paradigm might have induced substantial variation in connectivity 17 fluctuations over time (Garrett et al., 2013), which differs significantly from fluctuations 18 observed during resting-state. Such a possibility hampers the generalizability of our findings

19 to resting-state conditions. However, recently Betzel and colleagues (2016) showed that 20 during resting-state there is a number of periods during which manifest in- or decreases in 21 connectivity are present, and, in turn, influence the modular topology of resting-state 22 networks. In addition, the topography of resting-state networks not only can be obtained by 
Sliding-window analysis maps amygdala time-varying connectivity

1 temporal correlation of fMRI signals, but also by extracting brief moments when the regions

2 are transiently co-activated (Tagliazucchi et al. 2016; Liu and Duyn 2013). It is therefore

3 possible that task-induced co-activations persist in the subsequent resting-state period (Allan

4 et al. 2015), during which the dynamics of the amygdala connectivity observed in the current

5 study might be present as well. Future studies should directly investigate the relationship

6 between connectivity changes during resting and task conditions to better characterize temporal models of fluctuations in cognition and behavior over time.

Sliding-window analysis typically suffers from a relatively small number of data

9 points (for a typical TR of $2 \mathrm{~s}$ ), which might be overcome with recent technological advances

10 in fMRI acquisition. For example, multiband imaging (Feinberg et al., 2010; Moeller et al.,

11 2010) allow sub-second repetition times, and thereby provide an increase the number of sample points. This, in turn, increases the robustness of the correlation estimates, and enables better characterization of high-frequency components, which are mostly affected by nonneuronal noise such as cardiac and breathing rhythms. In addition to physiological noise, fMRI time-varying FC might be affected by variations in the BOLD signal mean and variance over time, hardware instability, or residual head motion. Though effects of the latter are highly debated in the functional connectivity community, a recent study demonstrated the existence of transient FC patterns in anesthetized monkeys (Hutchison et al., 2013b). Nevertheless, BOLD fluctuations are dominated by low frequencies, which makes fMRI particularly challenging to study connectivity dynamics. Yet, spectral characteristics of

21 BOLD activity reveal that resting-state networks are in fact broadband processes that span a wide frequency spectrum (Niazy et al., 2011), but are temporally blurred by the hemodynamic 
1 response function. Recently developed signal processing techniques, which are able to 2 temporally deconvolve fMRI time series, may prove helpful to use the full-spectrum of fMRI

3 signal, and hence provide better estimates of fMRI connectivity dynamics (e.g., Karahanoglu 4 and Van De Ville, 2015). Given that BOLD is an indirect measure of neuronal activity, it remains challenging to 6 elucidate whether fMRI time-varying FC represents dynamics of neuronal activity between 7 distant brain regions. Neuronal dynamics mostly have been investigated using electrophysiological recordings of single cells, local field potentials, and surface EEG (for a 9 review, see Rabinovich et al., 2012). Oscillations of electrical activity observed at different 10 frequencies enable populations of neurons to synchronize their activity over various time 11 scales (Friston and Buzsaki, 2016; Kiebel et al., 2008; Varela et al., 2001). Simultaneous EEG-fMRI studies have shown that BOLD connectivity variability partly reflects changes in neuronal synchrony associated with changes in vigilance states (Chang et al., 2013a; 14 Scheeringa et al., 2012; Tagliazucchi et al., 2012). Hence, dynamics of BOLD connectivity may emerge through transient synchrony in electrophysiological signals at multiple time 16 scales. We can only speculate that the fMRI connectivity fluctuations identified with SCL time series indeed stem from underlying neuronal dynamics. Future studies of simultaneous EEG-fMRI with a concurrently measured behavioral variable should shed more light on the neuronal sources of fMRI time-varying FC. 
Sliding-window analysis maps amygdala time-varying connectivity

2

3 Using sliding-window analysis, we demonstrate that fluctuations in left amygdala FC with

4 regions of the salience network were associated with transient changes in physiological 5 arousal and vigilance. These, in turn, were likely induced by a repetitive exposure to 6 unconditioned aversive stimuli (shocks) in our fear conditioning paradigm. The results of the 7 sliding-window analysis were demonstrated to be unlikely to have occurred by chance, were

16 This work was supported by the German Research Foundation (Deutsche 17 Forschungsgemeinschaft, DFG, FOR 1617: grant WA1539/7-1), and by a KOSMOS 18 Fellowship of the Humboldt Universität Berlin awarded to T.J. and H.W. (project nr. 19 01906020). We thank Dr. Hauke Heekeren for his insightful comments on an early version of 20 the article.

\section{Conclusions}

spatially distinct from static amygdala FC and from sliding-window FC of a control seed, but were highly comparable to the results of the PPI analysis. We conclude that sliding-window analysis can reveal functionally relevant connectivity fluctuations in the context of an externally cued task.

\section{Acknowledgments}


Sliding-window analysis maps amygdala time-varying connectivity

\section{Conflict of interest}

2

3 The authors declare no financial interests or potential conflicts of interest. 
Sliding-window analysis maps amygdala time-varying connectivity

\section{References}

Allan, T.W., Francis, S.T., Caballero-Gaudes, C., Morris, P.G., Liddle, E.B., Liddle, P.F., Brookes, M.J., Gowland, P.A., 2015. Functional Connectivity in MRI Is Driven by Spontaneous BOLD Events. PLoS One 10, e0124577.

Allen, E.A., Damaraju, E., Plis, S.M., Erhardt, E.B., Eichele, T., Calhoun, V.D., 2014. Tracking whole-brain connectivity dynamics in the resting state. Cereb Cortex 24, 663676.

Amaral, D.G., Price, J.L., 1984. Amygdalo-cortical projections in the monkey (Macaca fascicularis). J Comp Neurol 230, 465-496.

Angrilli, A., Mauri, A., Palomba, D., Flor, H., Birbaumer, N., Sartori, G., di Paola, F., 1996. Startle reflex and emotion modulation impairment after a right amygdala lesion. Brain 119 ( Pt 6), 1991-2000.

Bach, D.R., Flandin, G., Friston, K.J., Dolan, R.J., 2009. Time-series analysis for rapid eventrelated skin conductance responses. J Neurosci Methods 184, 224-234.

Baker, K.B., Kim, J.J., 2004. Amygdalar lateralization in fear conditioning: evidence for greater involvement of the right amygdala. Behav Neurosci 118, 15-23.

Barbas, H., De Olmos, J., 1990. Projections from the amygdala to basoventral and mediodorsal prefrontal regions in the rhesus monkey. J Comp Neurol 300, 549-571.

Barnett, A., 2016. URL: http://www.mathworks.com/matlabcentral/fileexchange/16062-testof-non-linearity/content/aaft.m

Betzel, R.F., Fukushima, M., He, Y., Zuo, X.N., Sporns, O., 2016. Dynamic fluctuations 
Sliding-window analysis maps amygdala time-varying connectivity

coincide with periods of high and low modularity in resting-state functional brain networks. Neuroimage 127, 287-297.

Bilkei-Gorzo, A., Erk, S., Schurmann, B., Mauer, D., Michel, K., Boecker, H., Scheef, L., Walter, H., Zimmer, A., 2012. Dynorphins regulate fear memory: from mice to men. J Neurosci 32, 9335-9343.

Birn, R.M., 2012. The role of physiological noise in resting-state functional connectivity. Neuroimage 62, 864-870.

Blakeslee, P., 1979. Attention and vigilance: performance and skin conductance response changes. Psychophysiology 16, 413-419.

Boucsein, W., 2012. Electrodermal Activity. Springer, New York.

Boucsein, W., Fowles, D.C., Grimnes, S., Ben-Shakhar, G., roth, W.T., Dawson, M.E., Filion, D.L., Society for Psychophysiological Research Ad Hoc Committee on Electrodermal, M., 2012. Publication recommendations for electrodermal measurements. Psychophysiology 49, 1017-1034.

Boveroux, P., Vanhaudenhuyse, A., Bruno, M.A., Noirhomme, Q., Lauwick, S., Luxen, A., Degueldre, C., Plenevaux, A., Schnakers, C., Phillips, C., Brichant, J.F., Bonhomme, V., Maquet, P., Greicius, M.D., Laureys, S., Boly, M., 2010. Breakdown of within- and between-network resting state functional magnetic resonance imaging connectivity during propofol-induced loss of consciousness. Anesthesiology 113, 1038-1053.

Bradley, M.M., Codispoti, M., Cuthbert, B.N., Lang, P.J., 2001. Emotion and motivation I: defensive and appetitive reactions in picture processing. Emotion 1, 276-298.

Chang, C., Cunningham, J.P., Glover, G.H., 2009. Influence of heart rate on the BOLD 
Sliding-window analysis maps amygdala time-varying connectivity

signal: the cardiac response function. Neuroimage 44, 857-869.

Chang, C., Glover, G.H., 2010. Time-frequency dynamics of resting-state brain connectivity measured with fMRI. Neuroimage 50, 81-98.

Chang, C., Liu, Z., Chen, M.C., Liu, X., Duyn, J.H., 2013a. EEG correlates of time-varying BOLD functional connectivity. Neuroimage 72, 227-236.

Chang, C., Metzger, C.D., Glover, G.H., Duyn, J.H., Heinze, H.J., Walter, M., 2013 b. Association between heart rate variability and fluctuations in resting-state functional connectivity. Neuroimage 68, 93-104.

Chen, A.C., Oathes, D.J., Chang, C., Bradley, T., Zhou, Z.W., Williams, L.M., Glover, G.H., Deisseroth, K., Etkin, A., 2013. Causal interactions between fronto-parietal central executive and default-mode networks in humans. Proc Natl Acad Sci U S A 110, 19944-19949.

Damaraju, E., Allen, E.A., Belger, A., Ford, J.M., McEwen, S., Mathalon, D.H., Mueller, B.A., Pearlson, G.D., Potkin, S.G., Preda, A., Turner, J.A., Vaidya, J.G., van Erp, T.G., Calhoun, V.D., 2014. Dynamic functional connectivity analysis reveals transient states of dysconnectivity in schizophrenia. Neuroimage Clin 5, 298-308.

Davis, M., 1992. The role of the amygdala in fear and anxiety. Annu Rev Neurosci 15, 353375.

Delgado, M.R., Olsson, A., Phelps, E.A., 2006. Extending animal models of fear conditioning to humans. Biol Psychol 73, 39-48.

Denny, B.T., Fan, J., Liu, X., Guerreri, S., Mayson, S.J., Rimsky, L., New, A.S., Siever, L.J., Koenigsberg, H.W., 2014. Insula-amygdala functional connectivity is correlated with 
Sliding-window analysis maps amygdala time-varying connectivity

habituation to repeated negative images. Soc Cogn Affect Neurosci 9, 1660-1667.

Etkin, A., Egner, T., Kalisch, R., 2011. Emotional processing in anterior cingulate and medial prefrontal cortex. Trends Cogn Sci 15, 85-93.

Fani, N., King, T.Z., Brewster, R., Srivastava, A., Stevens, J.S., Glover, E.M., Norrholm, S.D., Bradley, B., Ressler, K.J., Jovanovic, T., 2015. Fear-potentiated startle during extinction is associated with white matter microstructure and functional connectivity. Cortex 64, 249-259.

Feinberg, D.A., Moeller, S., Smith, S.M., Auerbach, E., Ramanna, S., Gunther, M., Glasser, M.F., Miller, K.L., Ugurbil, K., Yacoub, E., 2010. Multiplexed echo planar imaging for sub-second whole brain FMRI and fast diffusion imaging. PLoS One 5, e15710.

Feng, P., Feng, T., Chen, Z., Lei, X., 2014. Memory consolidation of fear conditioning: bistable amygdala connectivity with dorsal anterior cingulate and medial prefrontal cortex. Soc Cogn Affect Neurosci 9, 1730-1737.

F.I.N.D. Lab, 2016. URL: http://findlab.stanford.edu/functional_ROIs.html

Friston, K., Buzsaki, G., 2016. The Functional Anatomy of Time: What and When in the Brain. Trends Cogn Sci.

Friston, K.J., Buechel, C., Fink, G.R., Morris, J., Rolls, E., Dolan, R.J., 1997. Psychophysiological and modulatory interactions in neuroimaging. Neuroimage 6, 218229.

Frith, C.D., Allen, H.A., 1983. The skin conductance orienting response as an index of attention. Biol Psychol 17, 27-39.

Fullana, M.A., Harrison, B.J., Soriano-Mas, C., Vervliet, B., Cardoner, N., Àvila-Parcet, A., 
Sliding-window analysis maps amygdala time-varying connectivity

Radua, J., 2016. Neural signatures of human fear conditioning: an updated and extended meta-analysis of fMRI studies. Mol Psychiatry 21, 500-508.

Garcia-Larrea, L., Peyron, R., 2013. Pain matrices and neuropathic pain matrices: a review. Pain 154 Suppl 1, S29-43.

Garrett, D.D., Kovacevic, N., McIntosh, A.R., Grady, C.L., 2013. The modulation of BOLD variability between cognitive states varies by age and processing speed. Cereb Cortex 23, 684-693.

Ghashghaei, H.T., Barbas, H., 2002. Pathways for emotion: interactions of prefrontal and anterior temporal pathways in the amygdala of the rhesus monkey. Neuroscience 115 , 1261-1279.

Gianaros, P.J., Sheu, L.K., Matthews, K.A., Jennings, J.R., Manuck, S.B., Hariri, A.R., 2008. Individual differences in stressor-evoked blood pressure reactivity vary with activation, volume, and functional connectivity of the amygdala. J Neurosci 28, 990-999.

Gonzalez-Castillo, J., Handwerker, D.A., Robinson, M.E., Hoy, C.W., Buchanan, L.C., Saad, Z.S., Bandettini, P.A., 2014. The spatial structure of resting state connectivity stability on the scale of minutes. Front Neurosci 8, 138.

Gorgolewski, K.J., Varoquaux, G., Rivera, G., Schwarz, Y., Ghosh, S.S., Maumet, C., Sochat, V.V., Nichols, T.E., Poldrack, R.A., Poline, J.-B., Others, 2015. NeuroVault. org: a web-based repository for collecting and sharing unthresholded statistical maps of the human brain. Front Neuroinform 9, 8 .

Goulden, N., Khusnulina, A., Davis, N.J., Bracewell, R.M., Bokde, A.L., McNulty, J.P., Mullins, P.G., 2014. The salience network is responsible for switching between the 
Sliding-window analysis maps amygdala time-varying connectivity

default mode network and the central executive network: replication from DCM. Neuroimage 99, 180-190.

Greening, S.G., Mitchell, D.G.V., 2015. A network of amygdala connections predict individual differences in trait anxiety. Hum Brain Mapp 36, 4819-4830.

Handwerker, D.A., Roopchansingh, V., Gonzalez-Castillo, J., Bandettini, P.A., 2012. Periodic changes in fMRI connectivity. Neuroimage 63, 1712-1719.

Hindriks, R., Adhikari, M.H., Murayama, Y., Ganzetti, M., Mantini, D., Logothetis, N.K., Deco, G., 2015. Can sliding-window correlations reveal dynamic functional connectivity in resting-state fMRI? Neuroimage 127, 242-256.

Hlinka, J., Hadrava, M., 2015. On the danger of detecting network states in white noise. Front Comput Neurosci 9, 11.

Horovitz, S.G., Braun, A.R., Carr, W.S., Picchioni, D., Balkin, T.J., Fukunaga, M., Duyn, J.H., 2009. Decoupling of the brain's default mode network during deep sleep. Proc Natl Acad Sci U S A 106, 11376-11381.

Horovitz, S.G., Fukunaga, M., de Zwart, J.A., van Gelderen, P., Fulton, S.C., Balkin, T.J., Duyn, J.H., 2008. Low frequency BOLD fluctuations during resting wakefulness and light sleep: a simultaneous EEG-fMRI study. Hum Brain Mapp 29, 671-682.

Hutchison, R.M., Womelsdorf, T., Allen, E.A., Bandettini, P.A., Calhoun, V.D., Corbetta, M., Della Penna, S., Duyn, J.H., Glover, G.H., Gonzalez-Castillo, J., Handwerker, D.A., Keilholz, S., Kiviniemi, V., Leopold, D.A., de Pasquale, F., Sporns, O., Walter, M., Chang, C., 2013a. Dynamic functional connectivity: promise, issues, and interpretations. Neuroimage 80, 360-378. 
Sliding-window analysis maps amygdala time-varying connectivity

Hutchison, R.M., Womelsdorf, T., Gati, J.S., Everling, S., Menon, R.S., 2013b. Resting-state networks show dynamic functional connectivity in awake humans and anesthetized macaques. Hum Brain Mapp 34, 2154-2177.

Jenkinson, M., Bannister, P., Brady, M., Smith, S., 2002. Improved optimization for the robust and accurate linear registration and motion correction of brain images. Neuroimage 17, 825-841.

Ji, G., Neugebauer, V., 2009. Hemispheric lateralization of pain processing by amygdala neurons. J Neurophysiol 102, 2253-2264.

Jilka, S.R., Scott, G., Ham, T., Pickering, A., Bonnelle, V., Braga, R.M., Leech, R., Sharp, D.J., 2014. Damage to the Salience Network and interactions with the Default Mode Network. J Neurosci 34, 10798-10807.

Jones, D.T., Vemuri, P., Murphy, M.C., Gunter, J.L., Senjem, M.L., Machulda, M.M., Przybelski, S.A., Gregg, B.E., Kantarci, K., Knopman, D.S., Boeve, B.F., Petersen, R.C., Jack, C.R., Jr., 2012. Non-stationarity in the "resting brain's" modular architecture. PLoS One 7, e39731.

Karahanoglu, F.I., Van De Ville, D., 2015. Transient brain activity disentangles fMRI restingstate dynamics in terms of spatially and temporally overlapping networks. Nat Commun 6,7751 .

Kiebel, S.J., Daunizeau, J., Friston, K.J., 2008. A hierarchy of time-scales and the brain. PLoS Comput Biol 4, e1000209.

Kim, M.J., Loucks, R.A., Palmer, A.L., Brown, A.C., Solomon, K.M., Marchante, A.N., Whalen, P.J., 2011. The structural and functional connectivity of the amygdala: from 
Sliding-window analysis maps amygdala time-varying connectivity

normal emotion to pathological anxiety. Behav Brain Res 223, 403-410.

Kugiumtzis, D., 2000. Surrogate data test for nonlinearity including nonmonotonic transforms. Phys Rev E Stat Phys Plasmas Fluids Relat Interdiscip Topics 62, R25-28.

LaBar, K.S., Gatenby, J.C., Gore, J.C., LeDoux, J.E., Phelps, E.A., 1998. Human amygdala activation during conditioned fear acquisition and extinction: a mixed-trial fMRI study. Neuron 20, 937-945.

LeDoux, J.E., 2000. Emotion circuits in the brain. Annu Rev Neurosci 23, 155-184.

Leonardi, N., Van De Ville, D., 2015. On spurious and real fluctuations of dynamic functional connectivity during rest. Neuroimage 104, 430-436.

Lewis, C.M., Baldassarre, A., Committeri, G., Romani, G.L., Corbetta, M., 2009. Learning sculpts the spontaneous activity of the resting human brain. Proc Natl Acad Sci U S A $106,17558-17563$.

Liegeois, R., Ziegler, E., Phillips, C., Geurts, P., Gomez, F., Bahri, M.A., Yeo, B.T., Soddu, A., Vanhaudenhuyse, A., Laureys, S., Sepulchre, R., 2016. Cerebral functional connectivity periodically (de)synchronizes with anatomical constraints. Brain Struct Funct 221, 2985-2997.

Lim, C.L., Rennie, C., Barry, R.J., Bahramali, H., Lazzaro, I., Manor, B., Gordon, E., 1997. Decomposing skin conductance into tonic and phasic components. Int J Psychophysiol 25, 97-109.

Liu, C.C., Crone, N.E., Franaszczuk, P.J., Cheng, D.T., Schretlen, D.S., Lenz, F.A., 2011. Fear conditioning is associated with dynamic directed functional interactions between and within the human amygdala, hippocampus, and frontal lobe. Neuroscience 189, 
Sliding-window analysis maps amygdala time-varying connectivity

359-369.

Liu, X., Duyn, J.H., 2013. Time-varying functional network information extracted from brief instances of spontaneous brain activity. Proc Natl Acad Sci U S A 110, 4392-4397.

Moeller, S., Yacoub, E., Olman, C.A., Auerbach, E., Strupp, J., Harel, N., Ugurbil, K., 2010. Multiband multislice GE-EPI at 7 tesla, with 16-fold acceleration using partial parallel imaging with application to high spatial and temporal whole-brain fMRI. Magn Reson Med 63, 1144-1153.

Niazy, R.K., Xie, J., Miller, K., Beckmann, C.F., Smith, S.M., 2011. Spectral characteristics of resting state networks. Prog Brain Res 193, 259-276.

Phelps, E.A., Delgado, M.R., Nearing, K.I., LeDoux, J.E., 2004. Extinction learning in humans: role of the amygdala and vmPFC. Neuron 43, 897-905.

Phelps, E.A., LeDoux, J.E., 2005. Contributions of the amygdala to emotion processing: from animal models to human behavior. Neuron 48, 175-187.

Phelps, E.A., O’Connor, K.J., Gatenby, J.C., Gore, J.C., Grillon, C., Davis, M., 2001. Activation of the left amygdala to a cognitive representation of fear. Nat Neurosci 4, $437-441$.

Prado, J., Weissman, D.H., 2011. Heightened interactions between a key default-mode region and a key task-positive region are linked to suboptimal current performance but to enhanced future performance. Neuroimage 56, 2276-2282.

Preti, M.G., Bolton, T.A., Van De Ville, D., 2016. The dynamic functional connectome: State-of-the-art and perspectives. Neuroimage, doi:10.1016/j.neuroimage.2016.12.061.

Rabinovich, M.I., Friston, K.J., Varona, P., 2012. Principles of brain dynamics: Global state 
Sliding-window analysis maps amygdala time-varying connectivity

interactions. The MIT Press, Cambridge, MA.

Raz, G., Winetraub, Y., Jacob, Y., Kinreich, S., Maron-Katz, A., Shaham, G., Podlipsky, I., Gilam, G., Soreq, E., Hendler, T., 2012. Portraying emotions at their unfolding: a multilayered approach for probing dynamics of neural networks. Neuroimage 60, 14481461.

Roy, A.K., Shehzad, Z., Margulies, D.S., Kelly, A.M., Uddin, L.Q., Gotimer, K., Biswal, B.B., Castellanos, F.X., Milham, M.P., 2009. Functional connectivity of the human amygdala using resting state fMRI. Neuroimage 45, 614-626.

Sadaghiani, S., Kleinschmidt, A., 2013. Functional interactions between intrinsic brain activity and behavior. Neuroimage $80,379-386$.

Salimi-Khorshidi, G., Douaud, G., Beckmann, C.F., Glasser, M.F., Griffanti, L., Smith, S.M., 2014. Automatic denoising of functional MRI data: combining independent component analysis and hierarchical fusion of classifiers. Neuroimage 90, 449-468.

Scheeringa, R., Petersson, K.M., Kleinschmidt, A., Jensen, O., Bastiaansen, M.C., 2012. EEG alpha power modulation of fMRI resting-state connectivity. Brain Connect 2, 254-264.

Scholvinck, M.L., Maier, A., Ye, F.Q., Duyn, J.H., Leopold, D.A., 2010. Neural basis of global resting-state fMRI activity. Proc Natl Acad Sci U S A 107, 10238-10243.

Schultz, D.H., Balderston, N.L., Helmstetter, F.J., 2012. Resting-state connectivity of the amygdala is altered following Pavlovian fear conditioning. Front Hum Neurosci 6, 242.

Seeley, W.W., Menon, V., Schatzberg, A.F., Keller, J., Glover, G.H., Kenna, H., Reiss, A.L., Greicius, M.D., 2007. Dissociable intrinsic connectivity networks for salience processing and executive control. J Neurosci 27, 2349-2356. 
Sehlmeyer, C., Schoning, S., Zwitserlood, P., Pfleiderer, B., Kircher, T., Arolt, V., Konrad, C., 2009. Human fear conditioning and extinction in neuroimaging: a systematic review. PLoS One 4, e5865.

Shakil, S., Lee, C.H., Keilholz, S.D., 2016. Evaluation of sliding window correlation performance for characterizing dynamic functional connectivity and brain states. Neuroimage 133, 111-128.

Shehzad, Z., Kelly, A.M., Reiss, P.T., Gee, D.G., Gotimer, K., Uddin, L.Q., Lee, S.H., Margulies, D.S., Roy, A.K., Biswal, B.B., Petkova, E., Castellanos, F.X., Milham, M.P., 2009. The resting brain: unconstrained yet reliable. Cereb Cortex 19, 2209-2229.

Shen, K., Hutchison, R.M., Bezgin, G., Everling, S., McIntosh, A.R., 2015. Network structure shapes spontaneous functional connectivity dynamics. J Neurosci 35, 5579-5588.

Shirer, W.R., Ryali, S., Rykhlevskaia, E., Menon, V., Greicius, M.D., 2012. Decoding subject-driven cognitive states with whole-brain connectivity patterns. Cereb Cortex 22, 158-165.

Smith, S.M., Jenkinson, M., Woolrich, M.W., Beckmann, C.F., Behrens, T.E., Johansen-Berg, H., Bannister, P.R., De Luca, M., Drobnjak, I., Flitney, D.E., Niazy, R.K., Saunders, J., Vickers, J., Zhang, Y., De Stefano, N., Brady, J.M., Matthews, P.M., 2004. Advances in functional and structural MR image analysis and implementation as FSL. Neuroimage 23 Suppl 1, S208-219.

Smith, S.M., Miller, K.L., Moeller, S., Xu, J., Auerbach, E.J., Woolrich, M.W., Beckmann, C.F., Jenkinson, M., Andersson, J., Glasser, M.F., Van Essen, D.C., Feinberg, D.A., Yacoub, E.S., Ugurbil, K., 2012. Temporally-independent functional modes of 
Sliding-window analysis maps amygdala time-varying connectivity

spontaneous brain activity. Proc Natl Acad Sci U S A 109, 3131-3136.

Smith, S.M., Nichols, T.E., 2009. Threshold-free cluster enhancement: addressing problems of smoothing, threshold dependence and localisation in cluster inference. Neuroimage 44, 83-98.

Stein, M.B., Simmons, A.N., Feinstein, J.S., Paulus, M.P., 2007. Increased amygdala and insula activation during emotion processing in anxiety-prone subjects. Am J Psychiatry $164,318-327$.

Stein, J.L., Wiedholz, L.M., Bassett, D.S., Weinberger, D.R., Zink, C.F., Mattay, V.S., Meyer-Lindenberg, A., 2007. A validated network of effective amygdala connectivity. Neuroimage 36, 736-745.

Tagliazucchi, E., von Wegner, F., Morzelewski, A., Brodbeck, V., Laufs, H., 2012. Dynamic BOLD functional connectivity in humans and its electrophysiological correlates. Front Hum Neurosci 6, 339.

Tagliazucchi, E., Siniatchkin, M., Laufs, H., Chialvo, D.R., 2016. The Voxel-Wise Functional Connectome Can Be Efficiently Derived from Co-activations in a Sparse SpatioTemporal Point-Process. Front Neurosci 10, 381.

Tambini, A., Ketz, N., Davachi, L., 2010. Enhanced brain correlations during rest are related to memory for recent experiences. Neuron 65, 280-290.

van Olst, E.H., Orlebeke, J.F., Fokkema, S.D., 1967. Skin conductance as a measure of tonic and phasic arousal. Acta Psychol 27, 262.

van Well, S., Visser, R.M., Scholte, H.S., Kindt, M., 2012. Neural substrates of individual differences in human fear learning: evidence from concurrent fMRI, fear-potentiated 
Sliding-window analysis maps amygdala time-varying connectivity

startle, and US-expectancy data. Cogn Affect Behav Neurosci 12, 499-512.

Varela, F., Lachaux, J.P., Rodriguez, E., Martinerie, J., 2001. The brainweb: phase synchronization and large-scale integration. Nat Rev Neurosci 2, 229-239.

Veer, I.M., Oei, N.Y., Spinhoven, P., van Buchem, M.A., Elzinga, B.M., Rombouts, S.A., 2011. Beyond acute social stress: increased functional connectivity between amygdala and cortical midline structures. Neuroimage 57, 1534-1541.

Vytal, K.E., Overstreet, C., Charney, D.R., Robinson, O.J., Grillon, C., 2014. Sustained anxiety increases amygdala-dorsomedial prefrontal coupling: a mechanism for maintaining an anxious state in healthy adults. J Psychiatry Neurosci 39, 321-329.

Wager, T.D., Waugh, C.E., Lindquist, M., Noll, D.C., Fredrickson, B.L., Taylor, S.F., 2009. Brain mediators of cardiovascular responses to social threat: part I: Reciprocal dorsal and ventral sub-regions of the medial prefrontal cortex and heart-rate reactivity. Neuroimage 47, 821-835.

Wang, Z., Liu, J., Zhong, N., Qin, Y., Zhou, H., Li, K., 2012. Changes in the brain intrinsic organization in both on-task state and post-task resting state. Neuroimage 62, 394-407.

Wheelock, M.D., Sreenivasan, K.R., Wood, K.H., Ver Hoef, L.W., Deshpande, G., Knight, D.C., 2014. Threat-related learning relies on distinct dorsal prefrontal cortex network connectivity. Neuroimage 102, 904-912.

Winkler, A.M., Ridgway, G.R., Webster, M.A., Smith, S.M., Nichols, T.E., 2014. Permutation inference for the general linear model. Neuroimage 92, 381-397.

Yang, Z., Craddock, R.C., Margulies, D.S., Yan, C.G., Milham, M.P., 2014. Common intrinsic connectivity states among posteromedial cortex subdivisions: Insights from 
Sliding-window analysis maps amygdala time-varying connectivity

analysis of temporal dynamics. Neuroimage 93 Pt 1, 124-137.

Young, C.B., Raz, G., Everaerd, D., Beckmann, C.F., Tendolkar, I., Hendler, T., Fernández, G., Hermans, E.J., 2016. Dynamic shifts in large-scale brain network balance as a function of arousal. J Neurosci, doi:10.1523/jneurosci.1759-16.2016.

Zalesky, A., Breakspear, M., 2015. Towards a statistical test for functional connectivity dynamics. Neuroimage 114, 466-470.

Zalesky, A., Fornito, A., Cocchi, L., Gollo, L.L., Breakspear, M., 2014. Time-resolved resting-state brain networks. Proc Natl Acad Sci U S A 111, 10341-10346. 
Sliding-window analysis maps amygdala time-varying connectivity

Tables

Table 1. Regions that show a positive association between sliding-window functional connectivity of the left amygdala and fluctuations in skin conductance level.

\begin{tabular}{lcccccc}
\hline \multicolumn{1}{c}{ Region } & $\mathrm{L} / \mathrm{R}$ & $\begin{array}{c}\text { Number } \\
\text { of voxels }\end{array}$ & $\begin{array}{c}\text { Peak coordinates } \\
(\mathrm{MNI})\end{array}$ & $\mathrm{p}$-value \\
\hline Anterior Cingulate Gyrus & $\mathrm{R}$ & 4016 & 6 & 22 & 18 & .001 \\
Inferior Frontal Gyrus/ Pars & $\mathrm{R}$ & 510 & 56 & 10 & 2 & .004 \\
Opercularis & & & & & & \\
Cingulate Gyrus & $\mathrm{L}$ & 291 & -2 & -4 & 38 & .025 \\
Superior Frontal Gyrus & $\mathrm{R}$ & 56 & 22 & 46 & 24 & .042 \\
Brainstem & $\mathrm{L}$ & 50 & -4 & -14 & -10 & .017 \\
Supplementary Motor Cortex & $\mathrm{L}$ & 33 & -6 & 14 & 54 & .045 \\
Inferior Frontal Gyrus & $\mathrm{R}$ & 18 & 44 & 28 & 4 & .037 \\
Orbitofrontal Cortex & $\mathrm{R}$ & 16 & 24 & 30 & -14 & .033 \\
Thalamus & $\mathrm{R}$ & 15 & 16 & -10 & 14 & .039 \\
Brainstem & $\mathrm{R}$ & 11 & 12 & -8 & -12 & .037 \\
Postcentral Gyrus & $\mathrm{L}$ & 3 & -50 & -10 & 22 & .048 \\
Thalamus & $\mathrm{L}$ & 1 & -4 & -22 & -2 & .048 \\
\hline
\end{tabular}

Clusters are significant at $p<.05$, TFCE corrected for multiple comparisons. Anatomical labels of the peak voxel coordinates are identified with the Harvard-Oxford (Sub-)Cortical Structural Probability Atlas. R: right hemisphere; L: left hemisphere. 
Sliding-window analysis maps amygdala time-varying connectivity

Figures

Figure 1. First eigen-time-series of skin conductance level and amygdala-ACC slidingwindow connectivity across participants.

The time courses of the first eigen-time-series of (A) skin conductance level across the entire task scan, and (B) left amygdala-ACC sliding-window connectivity, which are most representative across the group of participants. The red vertical lines indicate the timing of electric shocks, for $1 \mathrm{a}$ in TRs, for $1 \mathrm{~b}$ in windows, where shocks are depicted at the window numbers in which shocks occurred exactly at the middle TR of the window.

Figure 2. Temporal association between fluctuations in functional connectivity and skin conductance level.

The statistical maps represent regions for which temporal fluctuations in functional connectivity of the left amygdala (panel A) and primary visual cortex (panel B), as obtained with the sliding-window analysis, show a positive association with fluctuations in skin conductance level. The voxelwise TFCE map is significant at $p<.05$, corrected for multiple comparisons, and overlaid on the $2 \mathrm{~mm}$ MNI template in radiological convention. Numbers in the bottom row indicate coordinates of the sagittal, coronal, and axial sections in MNI standard space. 
Figure 3. Temporal association between fluctuations in functional connectivity and skin conductance level using sliding-window and physio-physiological interaction (PPI) analyses.

The statistical maps represent regions for which temporal fluctuations in functional connectivity of the left amygdala show a positive association with fluctuations in skin conductance level, as obtained with either the sliding-window or PPI analysis. The voxelwise TFCE maps are reported for corrected $(p<.05)$ and uncorrected $(p<.01)$ thresholds for the sliding window and PPI analysis, respectively, and were overlaid on the $2 \mathrm{~mm}$ MNI template in radiological convention. Numbers in the bottom row indicate coordinates of the sagittal, coronal, and axial sections in MNI standard space.

\section{Supplementary material}

Figure S1. Seeds used in the functional connectivity analysis.

(A) Amygdala seeds were created with the Harvard-Oxford Subcortical Structural Probability Atlas (80\% probability).

(B) The anterior cingulate cortex (ACC) seed was created with a sphere of $6 \mathrm{~mm}$ radius, centered around a coordinate of peak significance in MNI standard space $[4,32,18]$.

(C) The primary visual cortex seed was obtained from the template of primary visual network (Functional Imaging in Neuropsychiatric Disorders [F.I.N.D.] Lab, 2016; Shirer et al., 2012), eroded by a $4 \mathrm{~mm}$-radius spherical kernel.

Figure S2. Group level mask of minimum whole brain coverage across all participants. 
Figure S3. Null distributions used in the surrogate analyses of sliding-window functional connectivity.

(A) Probability density of the standard deviation of the sliding-window functional connectivity under the null hypothesis. Functional connectivity was computed between the left amygdala and ACC ROI. The observed value was $0.265(p=.024)$, which is marked by the vertical red line.

(B) Probability density of the temporal association ( $t$ statistics) between skin conductance level and the sliding-window functional connectivity between the left amygdala and ACC ROI under the null hypothesis. The observed value was $3.74(p<.001)$, which is marked by the vertical red line.

Figure S4. Left amygdala functional connectivity across the whole task scan.

The statistical map represents regions that show the strongest positive temporal association with the left amygdala activity across the whole task scan. The FWE-corrected voxelwise map $(p<.05)$ was overlaid on the $2 \mathrm{~mm}$ MNI template in radiological convention. Numbers in the bottom row indicate coordinates of the sagittal, coronal, and axial sections in MNI standard space.

Figure S5. Association between SCL and fluctuations in BOLD activity across the whole task scan.

The statistical map represents regions for which fluctuations in BOLD activity are negatively associated with skin conductance level across the whole task scan. The voxelwise TFCE map 
Sliding-window analysis maps amygdala time-varying connectivity

is significant at $p<.05$, corrected for multiple comparisons, and overlaid on the $2 \mathrm{~mm} \mathrm{MNI}$ template in radiological convention. Numbers in the bottom row indicate coordinates of the sagittal, coronal, and axial sections in MNI standard space. 
A

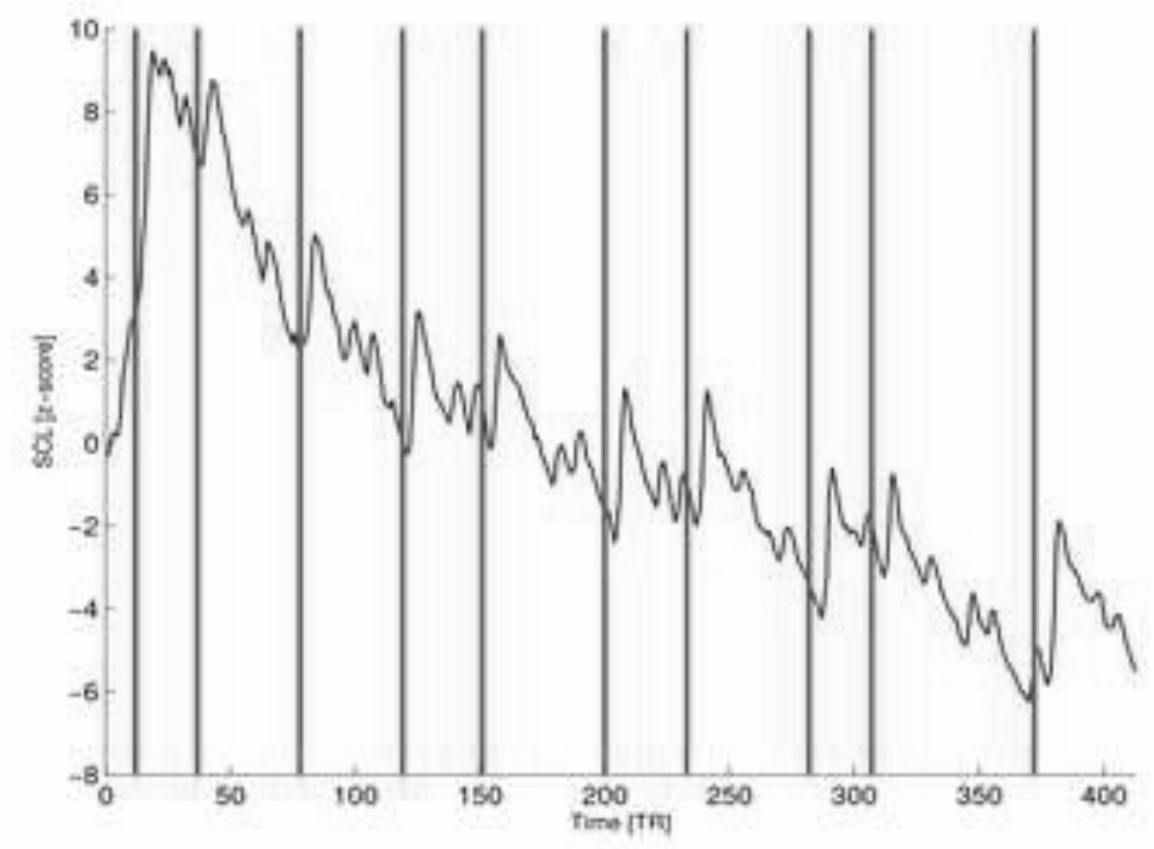

B

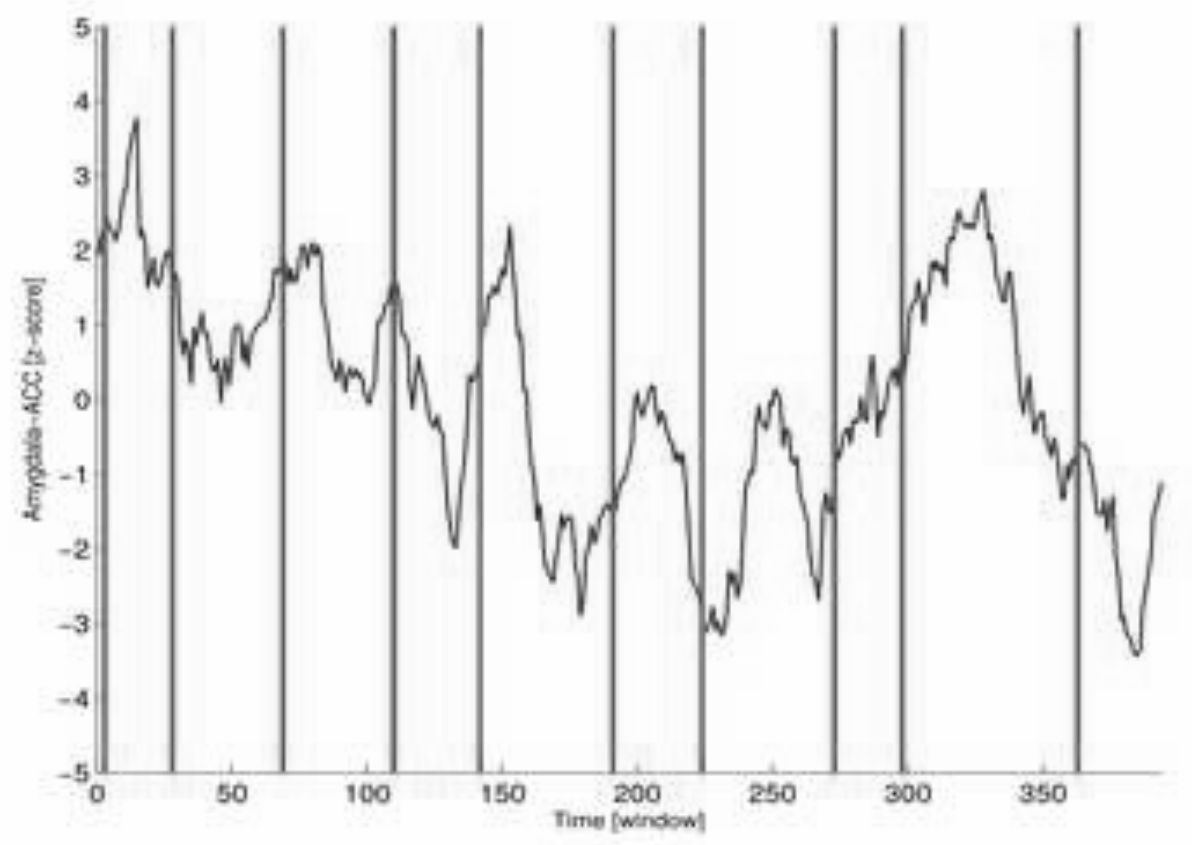


Panel A
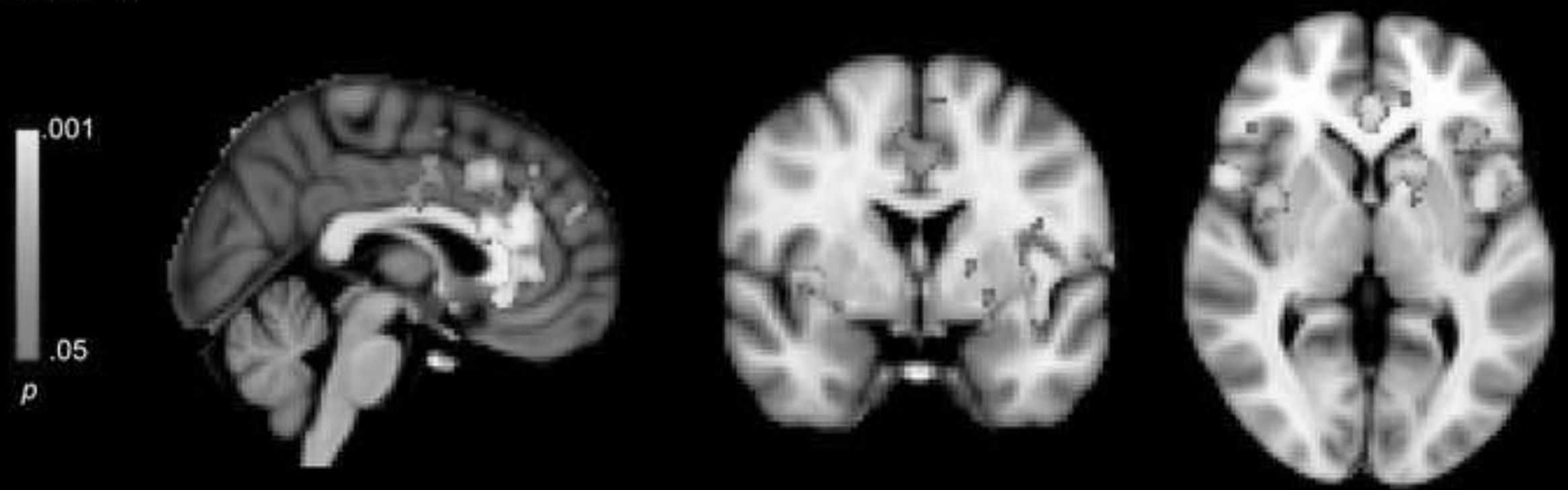

\section{Panel B}
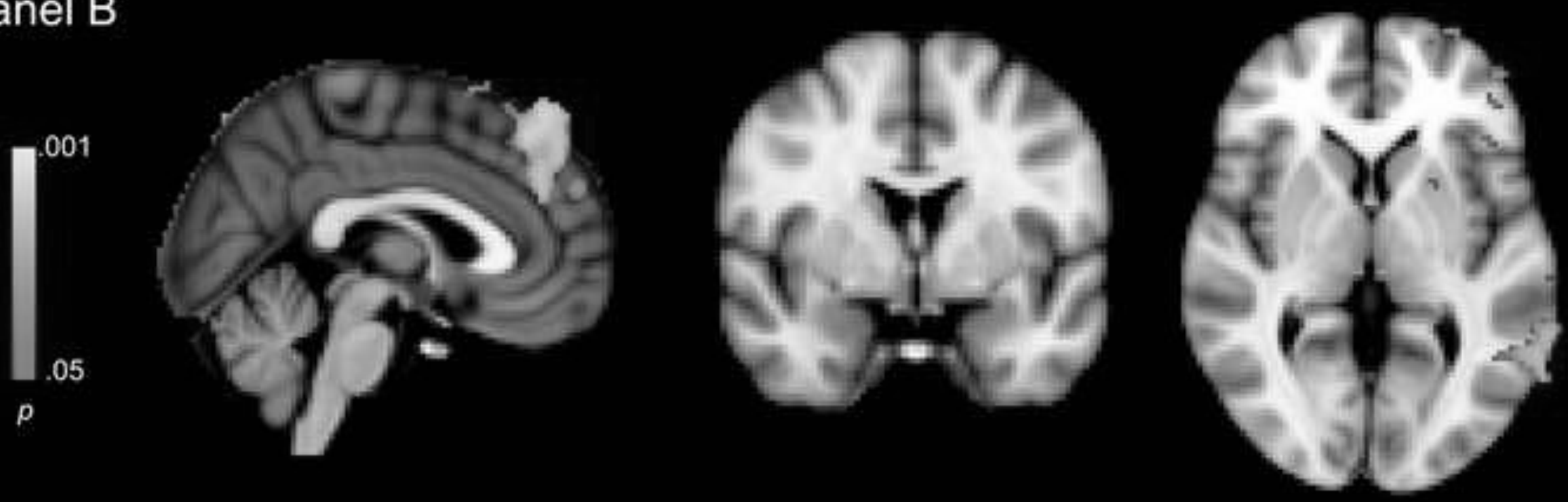


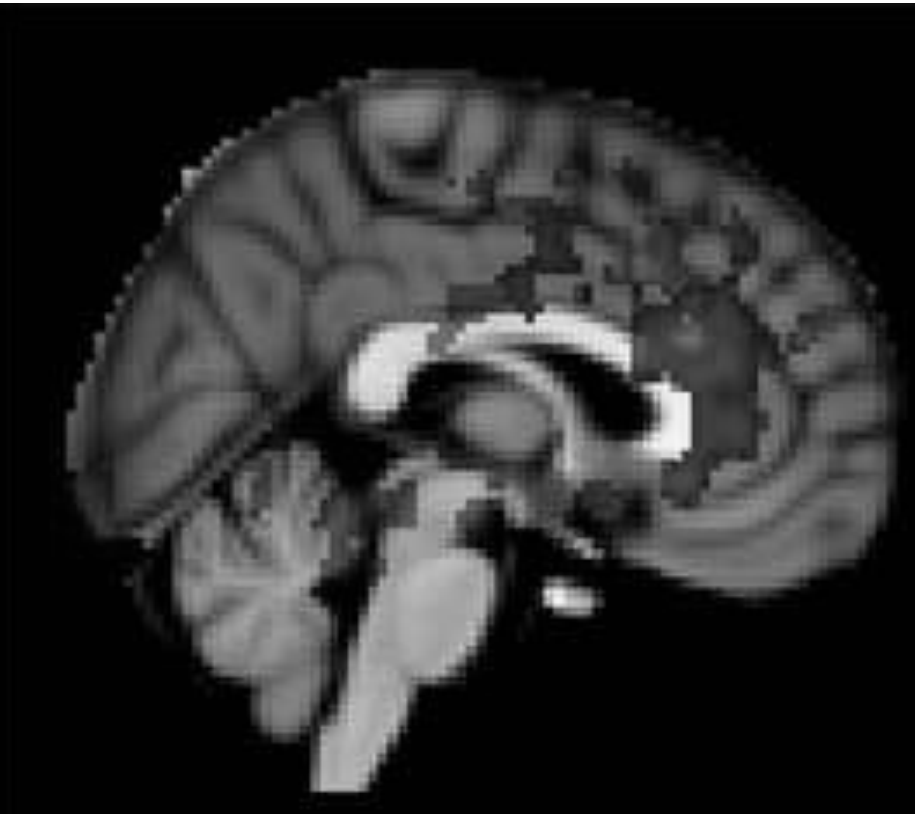

2

Sliding-window $p<.05$

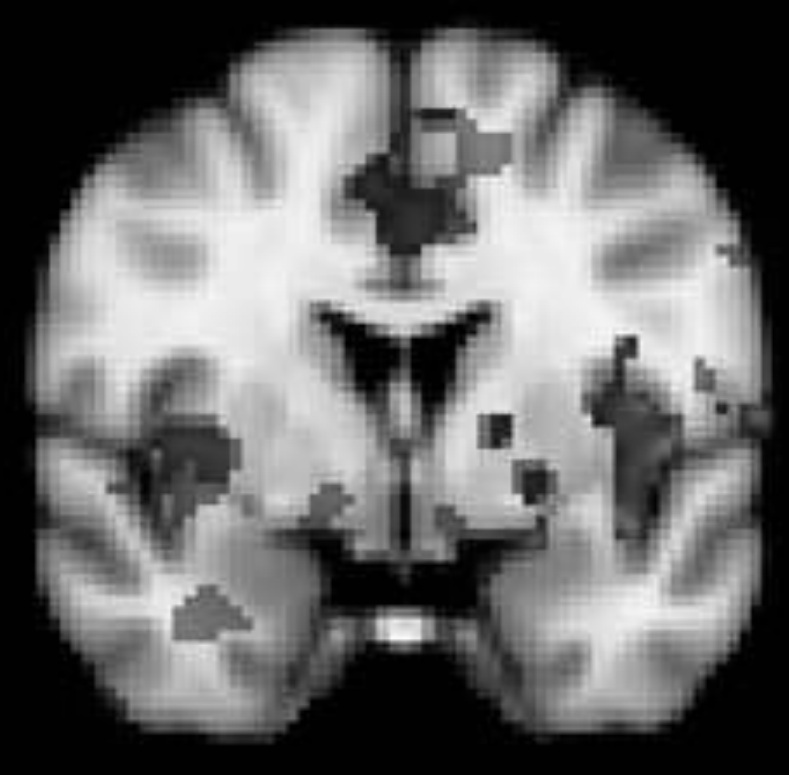

0

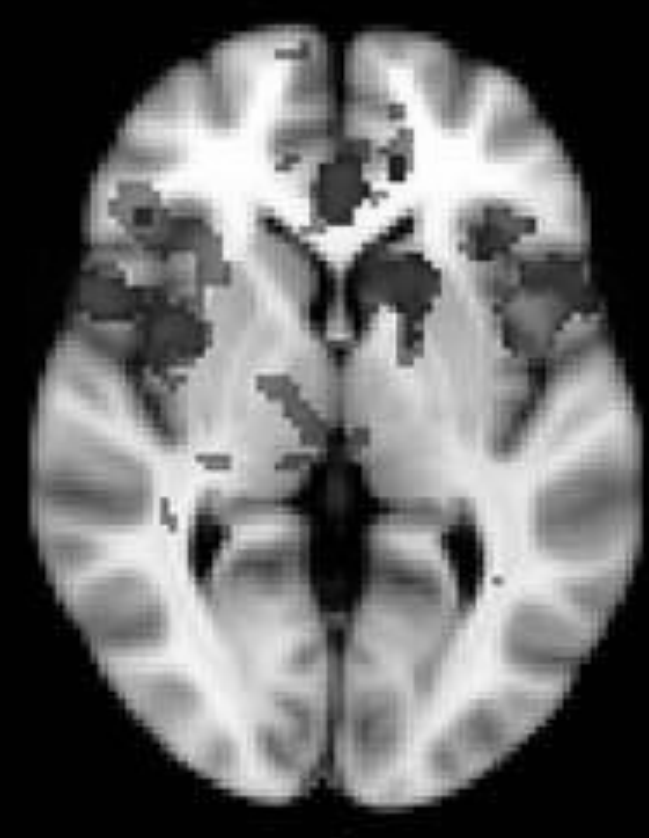

2
PPI

$p<.01$ uncorr.

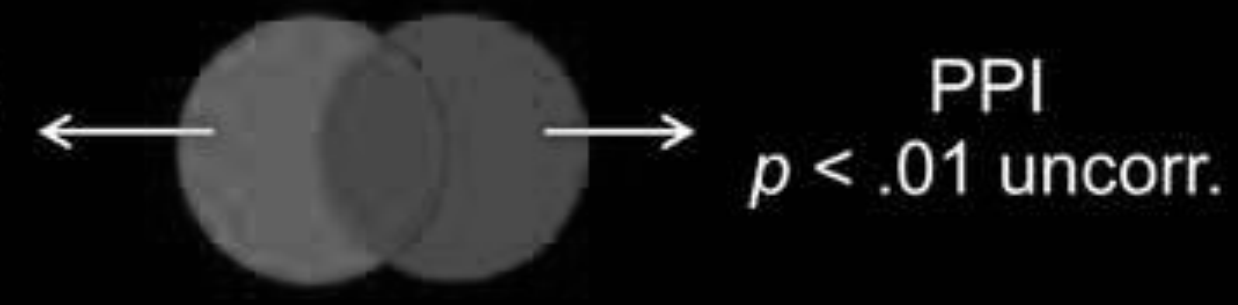


A

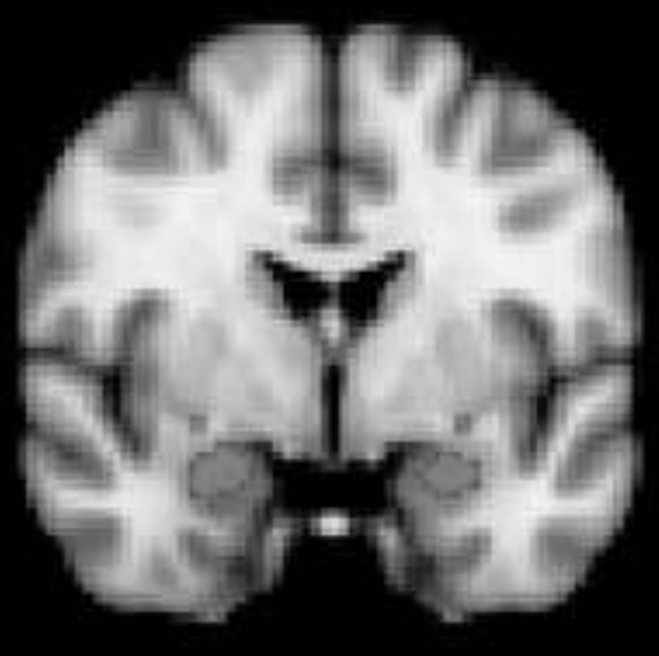

$-2$
B

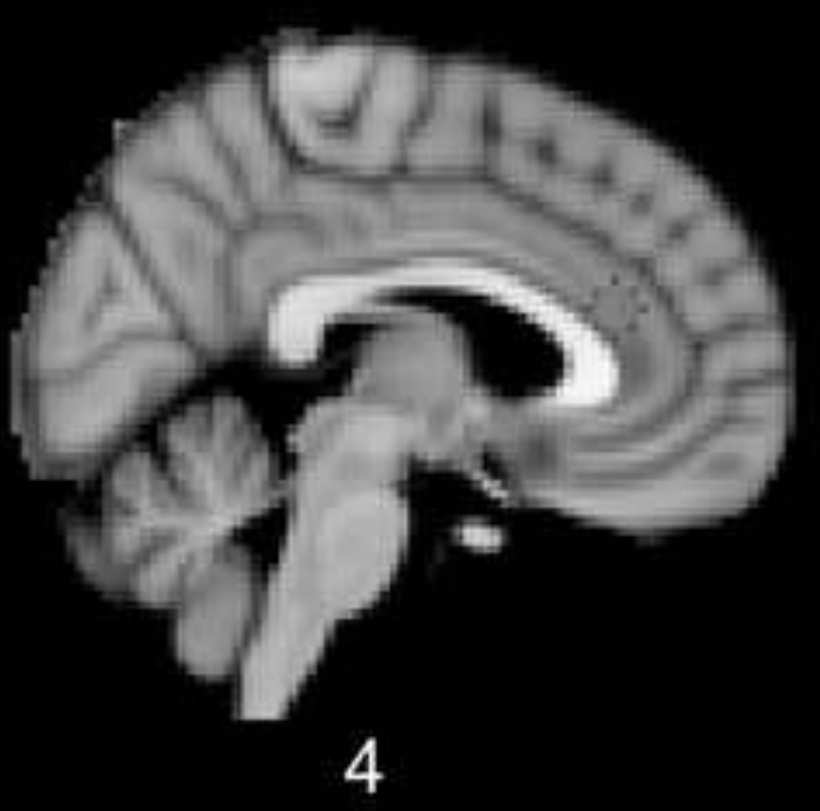

C

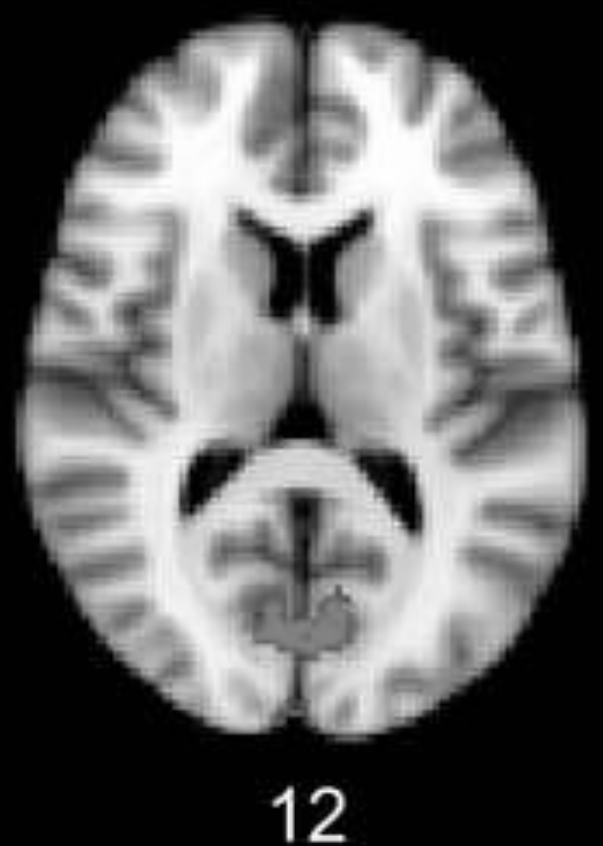


9. Supplemental Figure 2
Click here to download high resolution image

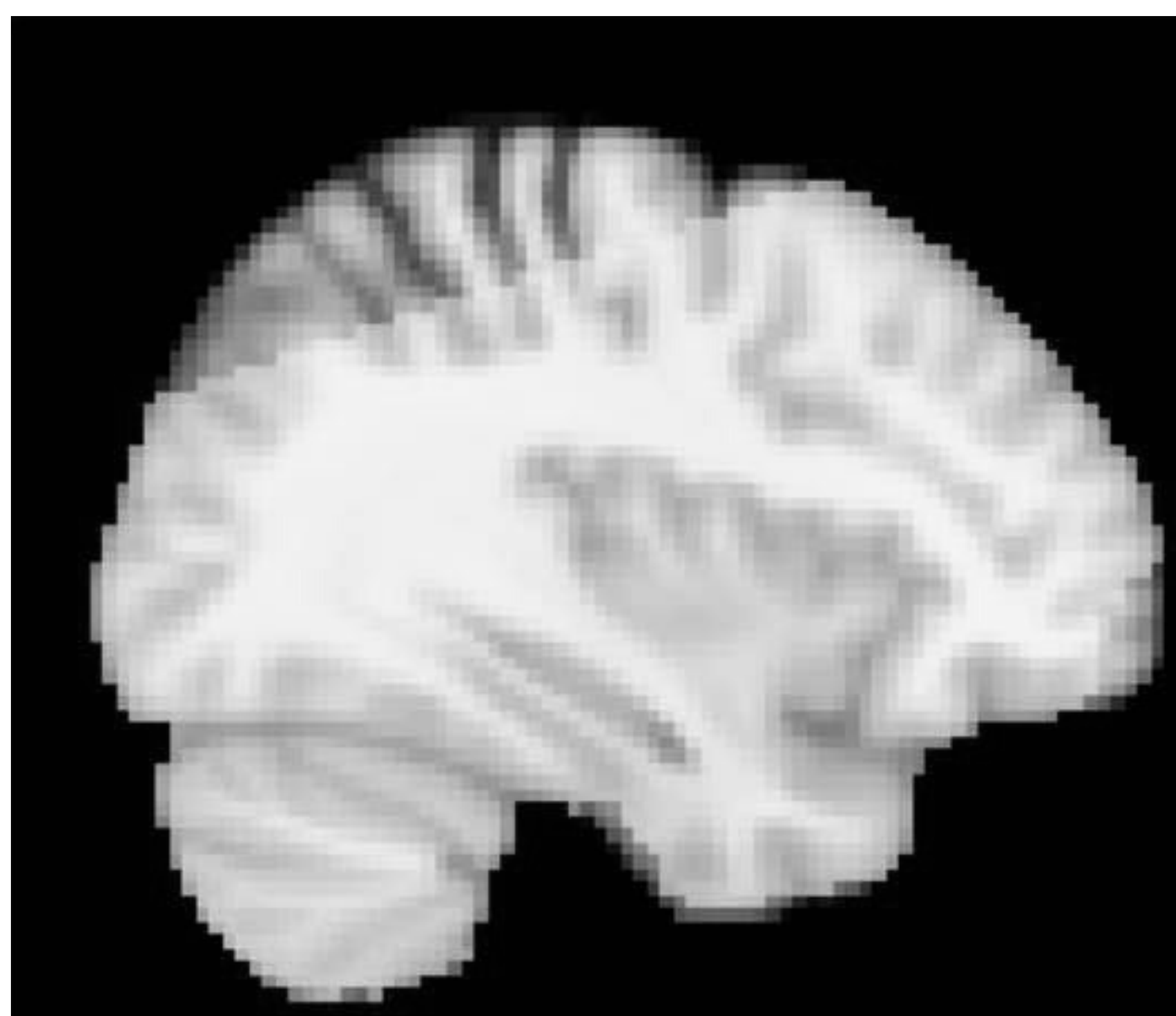

36 


\section{Supplemental Figure 3}

Click here to download high resolution image

A

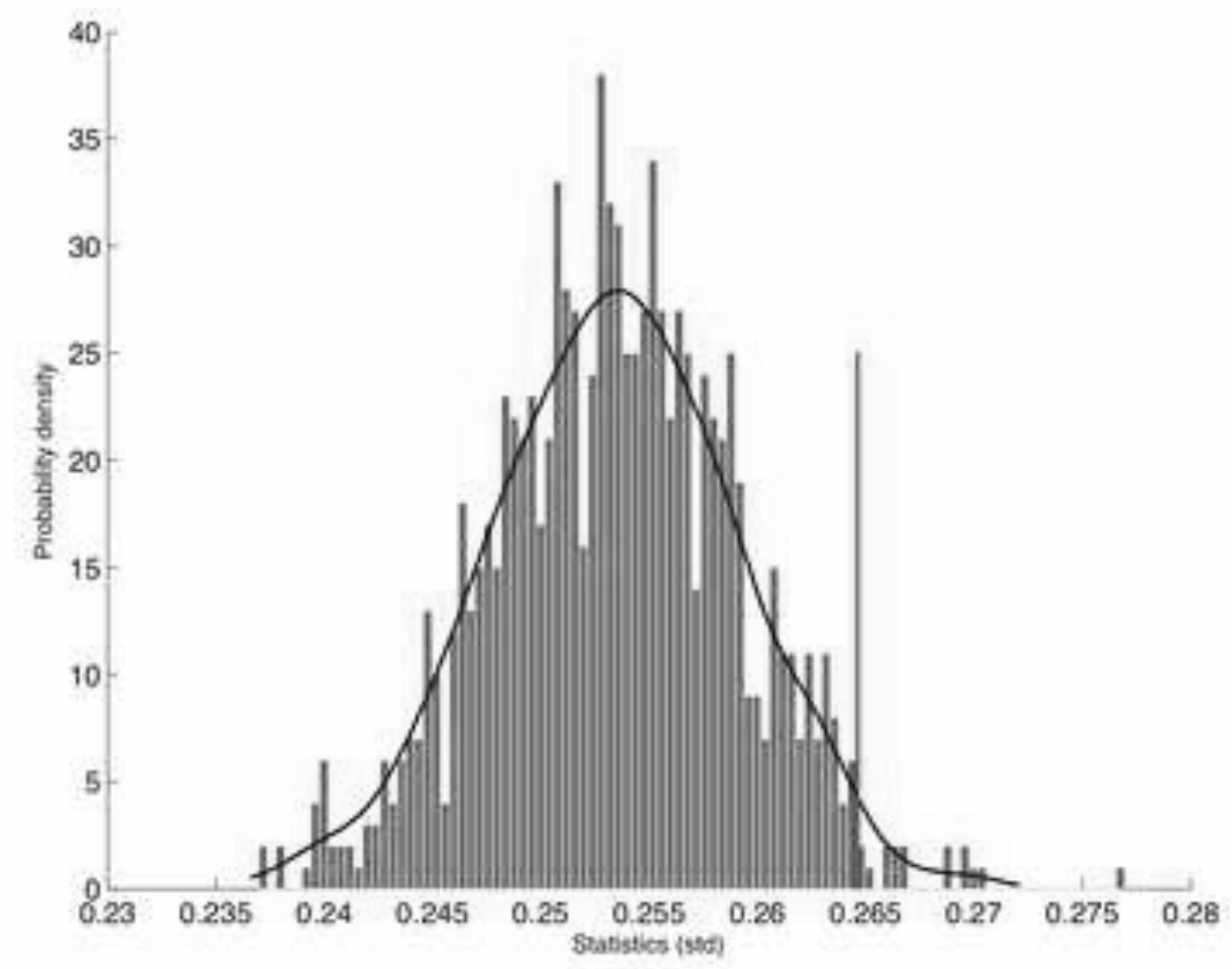

B

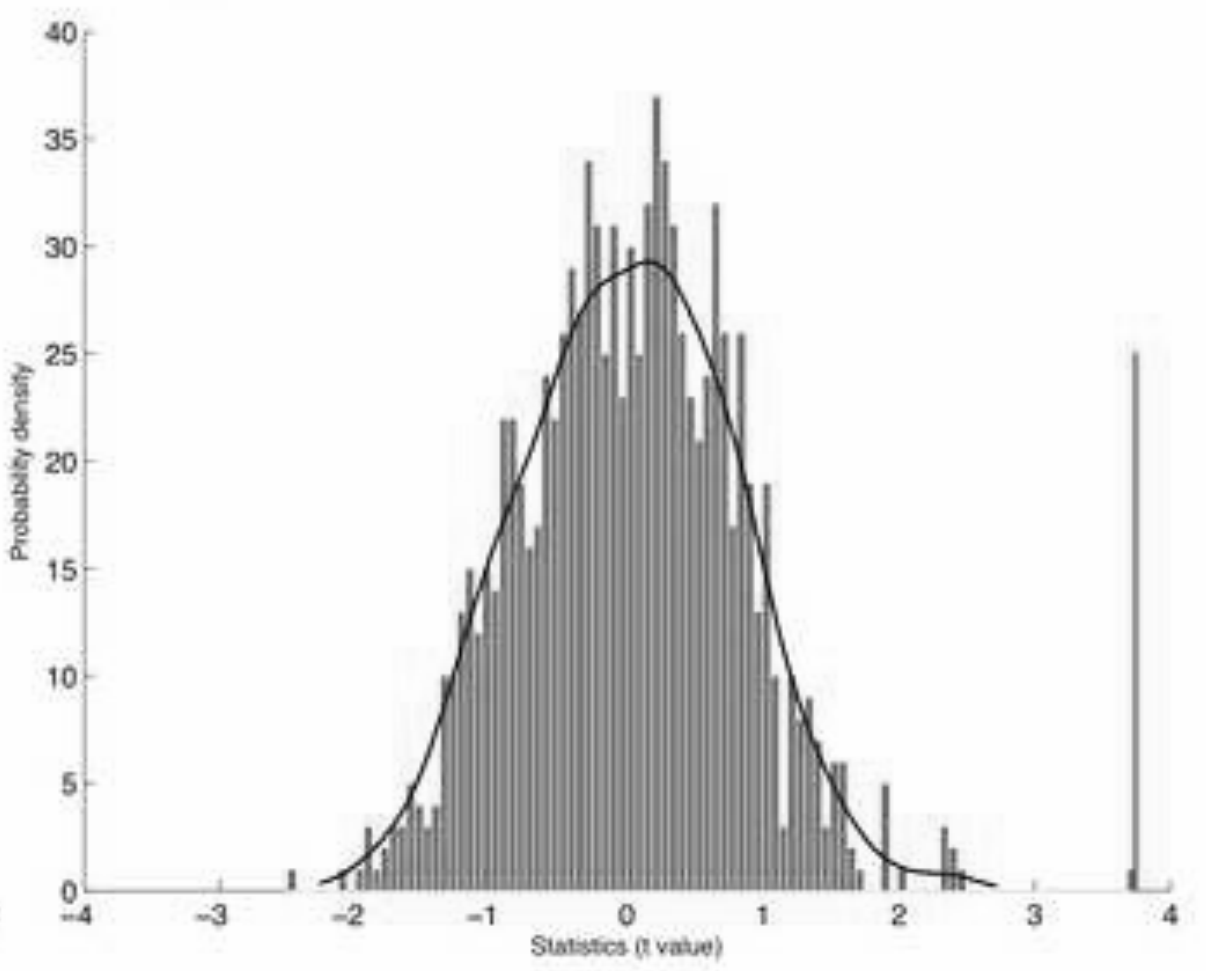




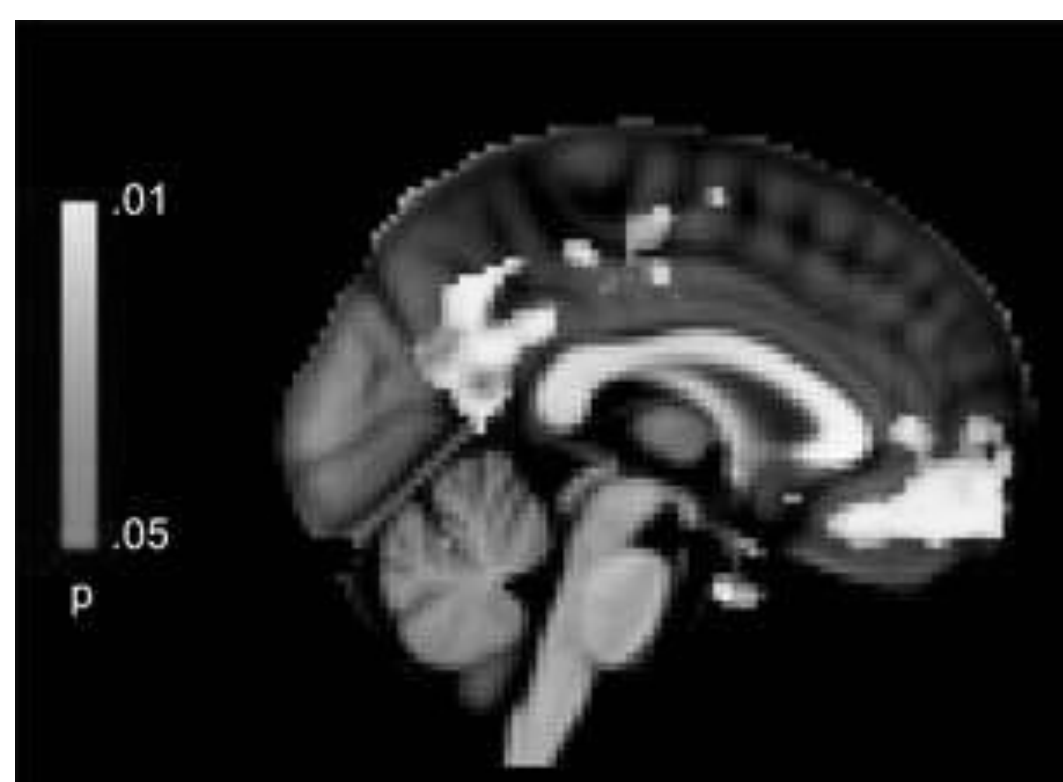

0

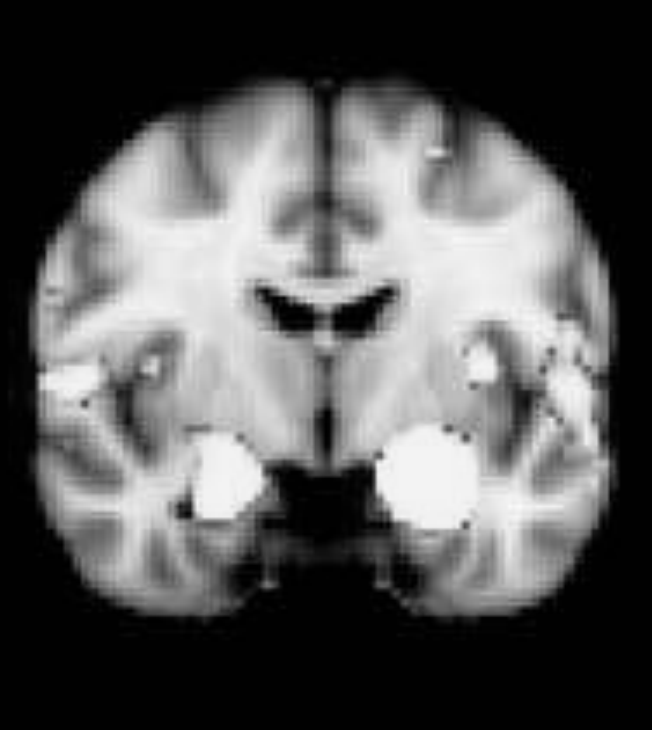

$-6$

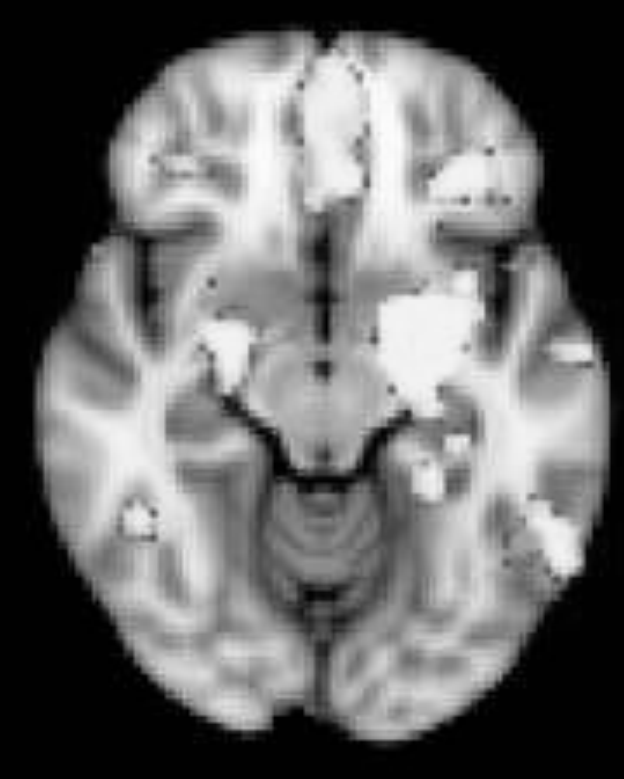

$-12$ 


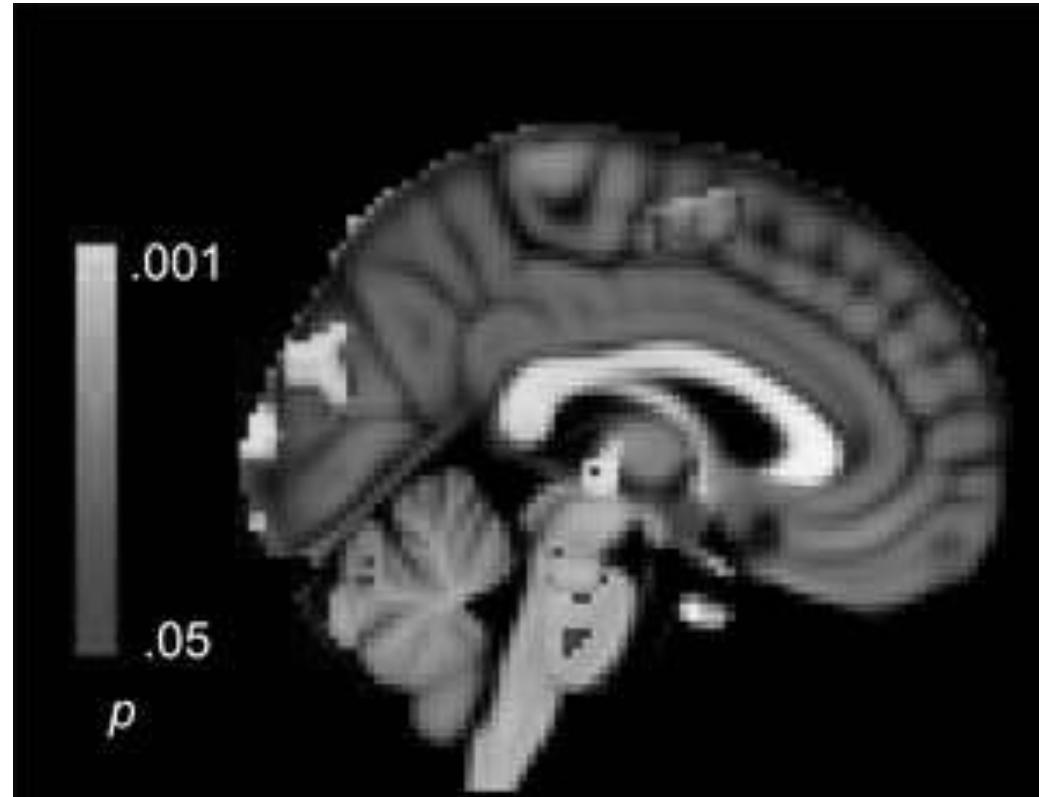

2

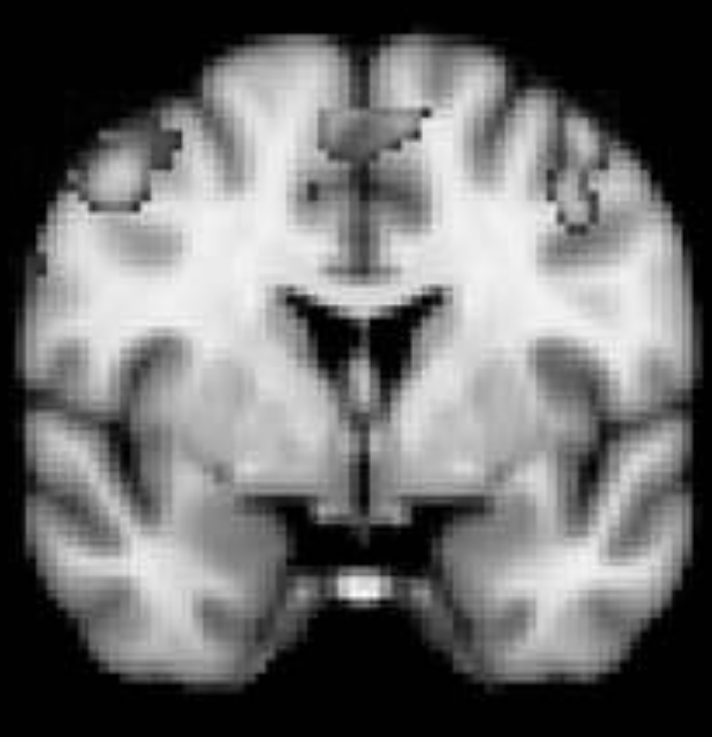

0

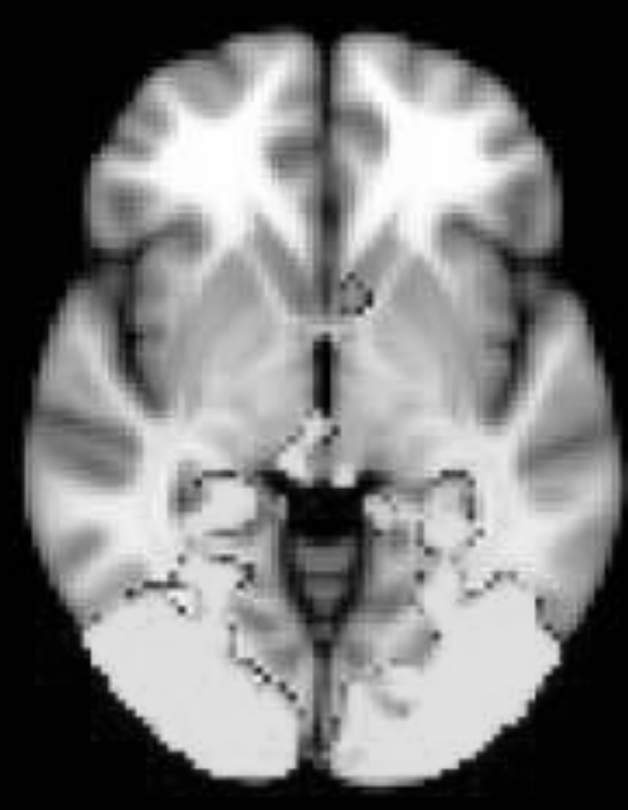

$-4$ 